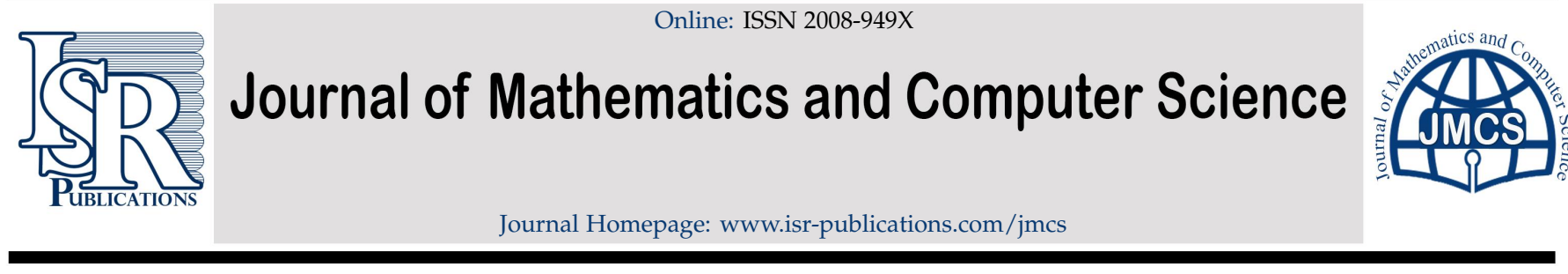

\title{
A novel three-level time-split approach for solving two-dimensional nonlinear unsteady convection-diffusion- reaction equation
}

\section{Eric Ngondiep}

Department of Mathematics and Statistics, College of Science, Imam Mohammad Ibn Saud Islamic University (IMSIU), 90950 Riyadh, Saudi Arabia.

Hydrological Research Centre, Institute for Geological and Mining Research, 4110 Yaounde, Cameroon.

\begin{abstract}
This paper considers a deep analysis of a three-level explicit time-split MacCormack method, namely the locally onedimensional explicit MacCormack for the numerical solution of the two-dimensional nonlinear evolutionary advection-diffusion equation subjects to suitable initial and boundary conditions. The splitting reduces the computational cost of the algorithm. Under a suitable time-step restriction, both theoretical and numerical results on the stability and error estimates of the scheme are deeply analyzed in $L^{m}\left(0, T ; L^{2}\right)$-norm $(m=1,2, \infty)$. The numerical experiments suggest that the proposed algorithm is easy to implement, temporal second-order convergent and fourth-order accurate in space. This shows the utility and efficiency of the considered method.
\end{abstract}

Keywords: 2D nonlinear unsteady advection-diffusion equation, one-dimensional operators (splitting), explicit MacCormack scheme, three-level explicit time-split MacCormack method, stability and convergence rate.

2020 MSC: 65M10, 65M05.

(C)2022 All rights reserved.

\section{Introduction and motivation}

This work considers a new numerical scheme for predicting the transport phenomena governed by the two-dimensional nonlinear time-dependent convection-diffusion-reaction equations. Such equations model a broad range of phenomenons in physical, chemical and biological sciences [2, 4, 31, 38, 41]. Furthermore, they arise in Helmholtz equation for modeling exterior acoustics, viscoelastic constitutive equations in modeling the extras stresses in non-Newtonian fluid flows and in a coupled magnetic/incompressible Navier-Stokes when computing the magnetic field [5, 9, 15]. For instance, chemical spills, groundwater contamination from leaky underground storage tanks, transport chemistry in the atmosphere or in combustion processes, turbulent flow modeling and active filtration or heat transport are serious problems. Although it is possible to assess the danger of the spread of the pollution released in the subsurface domain by the use of numerical simulations, it is worth noticing to mention that the

Email address: ericngondiep@gmail.com, engondiep@imamu.edu.sa (Eric Ngondiep)

doi: $10.22436 /$ jmcs.026.03.03

Received: 2021-08-12 Revised: 2021-09-02 Accepted: 2021-10-07 
ability to model unsteady flows and transport processes with complex physical interactions is particularly important from the point of view of physical realism, but is a difficult task. Therefore, the construction of accurate numerical methods for such problems in evolutionary cases presents a bigger challenge and less number of works on the subject appears in the literature (see $[6,7,13,34])$. In this paper, the governing problem for the two-dimensional nonlinear nonstationary convection-diffusion-reaction equation is

$$
\phi_{\mathrm{t}}+\mu \nabla \phi-\mathrm{a} \Delta \phi=f(\phi), \quad(x, y) \in \Omega, \quad \mathrm{t} \in(0, \mathrm{~T}],
$$

with the initial condition

$$
\phi(x, y, 0)=\phi_{0}(x, y), \quad(x, y) \in \bar{\Omega}
$$

and the boundary condition

$$
\phi(x, y, t)=\varphi(x, y, t), \quad(x, y) \in \partial \Omega, \quad t \in(0, T],
$$

where $\phi$ is the unknown quantity, $\mu$ is the given convective term, and $a>0$ represents the diffusive parameter, $f(\phi)$ denotes the nonlinear reaction term and $T>0$ is the final time. Furthermore, $\nabla$ and $\Delta$ are the gradient and the laplacian operators, respectively, $f \in \mathcal{C}^{1}(\mathbb{R})$ is a Lipschitz function, $\Omega=(0,1) \times(0,1)$ represents the fluid region, $\partial \Omega$ is the boundary of $\Omega$ and $\phi_{t}$ designates $\frac{\partial \phi}{\partial t}$. The initial condition $\phi_{0}$ and boundary condition $\varphi$ are assumed to be regular enough and satisfy the requirement $\varphi(x, y, 0)=\phi_{0}(x, y)$, for every $(x, y) \in \partial \Omega$, so that the initial-boundary value problem (1.1)-(1.3), admits a smooth solution (see for instance, $[12,17,43])$.

In the literature, the goal is to obtain approximate solutions to nonlinear parabolic equations (1.1)(1.3) using fast and efficient numerical schemes. Especially, one is interested in long term effects, so that the equations should be integrated over long time intervals. So, the use of some properties like the mass conservation, positivity and small phase errors must lead to numerical solutions which should be "qualitatively correct". Sometimes, the parabolic equations in a convection-dominated flow can be strong, local source terms. Thus, numerical algorithms are needed with good resolution of steep gradients. This note deals with the three-level explicit time-split MacCormack method which is too much fast and efficient than the two-step explicit MacCormack approach which in general, provides good resolutions of steep gradients.

During the last decades, the original MacCormack algorithm has been considered as one of the milestones of computational fluid dynamics [19-21,39]. This technique provides good resolutions of steep gradients. The first order time derivative is approximated at the predictor and corrector steps using forward difference representation with alternate one-side differentiating for the first order space derivatives. This is more convenient for systems of equations with nonlinear convective Jacobian matrices using second order one-side explicit methods, such as Lax-Wendroff technique [14, 23]. For high Reynolds numbers flows where the viscous regions are too thin, the method becomes very slow and can diverge. To overcome this difficulty, MacCormack develops a hybrid version of his scheme, the so called MacCormack rapid solver algorithm. The rapid solver method is a coupled of explicit MacCormack and an implicit scheme [18].

Most recently [24-30, 32, 33, 35-37, 40], the author applied the hybrid version of MacCormack and three-level time-split MacCormack approaches in a search of efficient solutions for the linear/nonlinear partial differential equations. More precisely, the rapid solver algorithm has been used for solving both mixed Stokes-Darcy and two-dimensional unsteady incompressible Navier-Stokes equations while the three-level time-split MacCormack was used in the numerical solutions of two-dimensional timedependent reaction-diffusion and coupled Burgers' equations. It is particularly important to recall that the three-level time-split applies to nonstationary equations of the form: $u_{t}=A_{1}(u)+A_{2}(u)$, where $u_{t}$ denotes $\frac{\partial u}{\partial t}$ and $A_{j}(j=1,2)$ are differential operators, so that the subproblems $u_{t}=A_{j}(u), j=1,2$, are each solved independently by the use of the explicit MacCormack method.

In $[3,8,11,22,42]$ several approaches for the numerical solutions of the evolutionary advectiondiffusion equation with constant coefficients are analyzed. The common idea in the considered problems 
is to carry out discretizations in two separable phases: partial discretization for the stabilized finite element procedures and temporal integration by time-marching finite difference representations. Such methods allow to combine various discretization schemes for the target model. Although some of these techniques have been very efficient (second order convergent in time and fourth order accurate in space), only the numerical studies have been considered in the literature. In the following, we analyze the threelevel time-split approach for the initial-boundary value problem (1.1)-(1.3). This study is motivated by: (a) a deep analysis of the theoretical and numerical results; (b) the method is fast, efficient and easy to implement (explicit, second order convergent in time and fourth order accurate in space); (c) the form of the time step requirement: $\max \left\{\frac{2 a k}{h^{2}}, \frac{|\mu| k}{h}\right\} \leqslant 1$, where $k$ is the time step and $h$ denotes the space step. Indeed, equations (1.1)-(1.3) can model nonlinear hyperbolic equations (case $a=0$ ) and parabolic problem (case $\mu=0$ ). For example, the time step restriction provided by the Fourier analysis for stability of explicit schemes when solving two-dimensional linear parabolic equations is given by $\frac{4 a k}{h^{2}} \leqslant 1$, which is well known in the literature as the CFL condition. Regarding the nonlinear convection-diffusion-reaction equations, the classical Von Neumann stability analysis is not in the standard sense, directly applicable; (d) it comes from item (c) that the solution is advanced in each direction with the maximum allowable time step. To explain this technique, we should consider the one-dimensional difference operators $L_{x}\left(k_{x}\right)$ and $L_{y}\left(k_{y}\right)$, where $k_{x}$ and $k_{x}$ denote the time steps in the $x$-direction and $y$-direction, respectively. The $\mathrm{L}_{x}\left(k_{x}\right)$ operator applied to $u_{i j}^{n}$,

$$
u_{i j}^{*}=L_{x}\left(k_{x}\right) u_{i j}^{n},
$$

is by definition equivalent to the two-step predictor-corrector MacCormack formulation. The $\mathrm{L}_{y}\left(k_{y}\right)$ operator is defined in a similar manner, that is,

$$
u_{i j}^{*}=L_{y}\left(k_{y}\right) u_{i j}^{n} .
$$

These expressions make use of a dummy time index, which is denoted by the asterisk. Setting $k_{x}=k$ and $k_{y}=\frac{k}{2 m}$, where $m$ is a positive integer, a high order convergent scheme can be constructed by applying the $L_{x}$ and $L y$ operators to $u_{i j}^{n}$, in the following manner

$$
u_{i j}^{n+1}=\left[L_{y}\left(\frac{k}{2 m}\right)\right]^{m} L_{x}(k)\left[L_{y}\left(\frac{k}{2 m}\right)\right]^{m} u_{i j}^{n} .
$$

In the case where $h_{y}<<h_{x},\left(h_{x}\right.$ and $h_{y}$ are the mesh sizes in the $x$-direction and $y$-direction, respectively), we get interesting numerical schemes.

In the following, we consider the 1D difference operators $L_{x}\left(k_{x}\right)$ and $L_{y}\left(k_{y}\right)$ defined by equations (1.4) and (1.5), respectively. Specifically, we set $m=1$ and a second-order accurate scheme can be constructed by applying the $L_{x}$ and $L_{y}$ operators to $u_{i j}^{n}$ as follows

$$
u_{i j}^{n+1}=L_{y}(k / 2) L_{x}(k) L_{y}(k / 2) u_{i j}^{n} .
$$

Letting $k_{x}=k, k_{y}=\frac{k}{2}$ and $h_{x}=h_{y}:=h$, it comes from equations (1.4), (1.5), and (1.6) that

$$
u_{i j}^{*}=L_{y}(k / 2) u_{i j}^{n}, u_{i j}^{* *}=L_{x}(k) u_{i j}^{*}=L_{x}(k) L_{y}(k / 2) u_{i j}^{n} \text { and } u_{i j}^{n+1}=L_{y}(k / 2) u_{i j}^{* *} .
$$

To construct our algorithm, we should find explicit expressions of equations $u_{i j}^{*}=L_{y}(k / 2) u_{i j}^{n}$ and $u_{i j}^{* *}=L_{x}(k) u_{i j}^{*}$. This will allow to provide an explicit formula of the equation $u_{i j}^{n+1}=L_{y}(k / 2) u_{i j}^{* *}$, which represents a "one-step time-split MacCormack algorithm". For the sake of simplicity, we use notations: $u)_{i j}^{n}=u_{i j}^{n}$ and $[u+v]_{i j}^{n}=u_{i j}^{n}+v_{i j}^{n}$.

The goal of this work is to analyze the following items:

1. full description of a three-level time-split explicit MacCormack scheme for solving the nonlinear time-dependent convection-diffusion-reaction problem (1.1)-(1.3); 
2. stability analysis of the method;

3. error estimates of the numerical scheme;

4. numerical experiments which provide the convergence rate, confirms the theoretical analysis and shows the efficiency and effectiveness of the algorithm.

Items 1-4, represent our original contributions since as far as we know, there is no available works in literature which solves the nonlinear convection-diffusion-reaction model (1.1)-(1.3), using a time-split MacCormack method.

The paper is organized as follows. In Section 2, we present a detailed description of the three-level time-split MacCormack method for solving the initial-boundary value problem (1.1)-(1.3). Section 3 studies the stability of the numerical scheme under the time-step restriction given above, while Section 4 considers a deep analysis of the error estimates and the convergence of the method. A wide set of numerical examples which provide the convergence rate of the new algorithm and confirms the theoretical result (on the stability) are presented and discussed in Section 5. We draw the conclusion and present our future direction of works in Section 6.

\section{Full description of the numerical scheme}

This section considers a full description a three-level time-split MacCormack scheme for solving the two-dimensional nonlinear time-dependent convection-diffusion-reaction equations (1.1)-(1.3).

Let $N$ and $M$ be two positive integers. Set $k:=\Delta t=\frac{T}{N}, h:=\Delta x=\Delta y=\frac{1}{M}$, be the time step and mesh size, respectively. Let $t^{n}=k n$, be the time used at the first step, $t^{*}$ be the starting time at the second phase, $t^{*} \in\left(t^{n}, t^{n+1}\right)$ and $t^{* *}$ be the time considered at the third stage in a three-level time-split MacCormack, $t^{* *} \in\left(t^{*}, t^{n+1}\right)$, for $n=0,1,2, \ldots, N-1, x_{i}=i h, y_{j}=j h$, for $0 \leqslant i, j \leqslant M$. Furthermore, we define the discrete regions: $\Omega_{k}=\left\{t^{n}, 0 \leqslant n \leqslant N\right\}, \bar{\Omega}_{h}=\left\{\left(x_{i}, y_{j}\right), 0 \leqslant i, j \leqslant M\right\}, \Omega_{h}=\bar{\Omega}_{h} \cap \Omega$ and $\partial \Omega_{\mathrm{h}}=\bar{\Omega}_{\mathrm{h}} \cap \partial \Omega$.

Suppose $\mathcal{U}_{h}=\left\{\phi_{i j}^{n}=\phi\left(x^{i}, y^{j}, t^{n}\right), n=0,1, \ldots, N ; 0 \leqslant i, j \leqslant M\right\}$, be the space of grid functions defined on $\Omega_{h} \times \Omega_{k}$. Set

$$
\begin{array}{rlrl}
\delta_{t} \phi_{i j}^{*} & =\frac{\phi_{i j}^{*}-\phi_{i j}^{n}}{k / 2}, & \delta_{t} \phi_{i j}^{* *}=\frac{\phi_{i j}^{* *}-\phi_{i j}^{*}}{k}, & \delta_{t} \phi_{i j}^{n+1}=\frac{\phi_{i j}^{n+1}-\phi_{i j}^{* *}}{k / 2}, \\
\delta_{x} \phi_{i+\frac{1}{2}, j}^{n}=\frac{\phi_{i+1, j}^{n}-\phi_{i j}^{n}}{h}, & \delta^{x} \phi_{i j}^{n}=\frac{\phi_{i+1, j}^{n}-\phi_{i-1, j}^{n}}{2 h}, & \delta_{y} \phi_{i, j+\frac{1}{2}}^{n}=\frac{\phi_{i, j+1}^{n}-\phi_{i j}^{n}}{h}, \\
\delta^{y} \phi_{i j}^{n} & =\frac{\phi_{i, j+1}^{n}-\phi_{i, j-1}^{n}}{2 h}, & \delta_{x}^{2} \phi_{i j}^{n}=\frac{\delta_{x} \phi_{i+\frac{1}{2}, j}^{n}-\delta_{x} \phi_{i-\frac{1}{2}, j}^{n}}{h}, & \delta_{y}^{2} \phi_{i j}^{n}=\frac{\delta_{y} \phi_{i, j+\frac{1}{2}}^{n}-\delta_{y} \phi_{i, j-\frac{1}{2}}^{n}}{h} .
\end{array}
$$

From the definition of the linear operators $\delta^{x}$ and $\delta^{y}$, it is easy to see that $\delta^{x} \phi_{i j}^{n}=\frac{1}{2}\left(\delta_{x} \phi_{i+\frac{1}{2}, j}^{n}+\delta_{x} \phi_{i-\frac{1}{2}, j}^{n}\right)$ and $\delta^{y} \phi_{i j}^{n}=\frac{1}{2}\left(\delta_{y} \phi_{i, j+\frac{1}{2}}^{n}+\delta_{y} \phi_{i, j-\frac{1}{2}}^{n}\right)$. The discrete norms are defined as follows

$$
\begin{gathered}
\left\|\phi^{\mathrm{n}}\right\|_{\mathrm{L}^{2}}=\mathrm{h}\left(\sum_{i, j=1}^{\mathrm{M}-1}\left|\phi_{i j}^{\mathrm{n}}\right|^{2}\right)^{\frac{1}{2}}, \quad\left\|\delta_{x} \phi^{\mathrm{n}}\right\|_{\mathrm{L}^{2}}=h\left(\sum_{j=1}^{\mathrm{M}-1} \sum_{i=0}^{M-1}\left|\delta_{x} \phi_{i+\frac{1}{2}, j}^{\mathrm{n}}\right|^{2}\right)^{\frac{1}{2}}, \\
\left\|\delta_{y} \phi^{\mathrm{n}}\right\|_{\mathrm{L}^{2}}=\mathrm{h}\left(\sum_{j=0}^{\mathrm{M}-1} \sum_{i=1}^{M-1}\left|\delta_{y} \phi_{i, j+\frac{1}{2}}^{\mathrm{n}}\right|^{2}\right)^{\frac{1}{2}}, \quad\left\|\delta_{\lambda}^{2} \phi^{\mathrm{n}}\right\|_{\mathrm{L}^{2}}=\mathrm{h}\left(\sum_{i, j=1}^{\mathrm{M}-1}\left|\delta_{\lambda}^{2} \phi_{i j}^{\mathrm{n}}\right|^{2}\right)^{\frac{1}{2}},
\end{gathered}
$$

where $\lambda=x$ or $y$. Furthermore, the scalar products are defined by

$$
\left(\phi^{n}, v^{n}\right)=h^{2} \sum_{i, j=1}^{M-1} \phi_{i j}^{n} v_{i j}^{n}, \quad<\delta_{x} \phi^{n}, \delta_{x} v^{n}>_{x}=h^{2} \sum_{j=1}^{M-1} \sum_{i=0}^{M-1} \delta_{x} \phi_{i+\frac{1}{2}, j}^{n} \delta_{x} v_{i+\frac{1}{2}, j^{\prime}}^{n}
$$


and

$$
<\delta_{y} \phi^{n}, \delta_{y} v^{n}>_{y}=h^{2} \sum_{j=0}^{M-1 M-1} \sum_{i=1}^{M-1} \delta_{y} \phi_{i, j+\frac{1}{2}}^{n} \delta_{y} v_{i, j+\frac{1}{2}}^{n} .
$$

The sobolev space $H^{1}(\Omega)$ endowed with the norm $|\cdot|_{H^{1}}$ (respectively, $\|\cdot\|_{H^{1}}$ ) is defined as

$$
\left|\phi^{\mathrm{n}}\right|_{\mathrm{H}^{1}}=\left(\left\|\delta_{x} \phi^{\mathrm{n}}\right\|_{\mathrm{L}^{2}}^{2}+\left\|\delta_{y} \phi^{\mathrm{n}}\right\|_{\mathrm{L}^{2}}^{2}\right)^{\frac{1}{2}} \text { and }\left\|\phi^{\mathrm{n}}\right\|_{\mathrm{H}^{1}}=\left(\left\|\phi^{\mathrm{n}}\right\|_{\mathrm{L}^{2}}^{2}+\left\|\delta_{x} \phi^{\mathrm{n}}\right\|_{\mathrm{L}^{2}}^{2}+\left\|\delta_{y} \phi^{\mathrm{n}}\right\|_{\mathrm{L}^{2}}^{2}\right)^{\frac{1}{2}} \text {. }
$$

It is worth noticing to recall that a time-split MacCormack [27, 32] splits the two-step explicit MacCormack method into a sequence of one-dimensional operations, thereby achieving a good stability condition. More precisely, the splitting makes it possible to advance the solution in each direction with the maximum allowable time step ([1], page 231).

Applying the Taylor series expansion for $\phi$ about $\left(x_{i}, y_{j}, t^{n}\right)$ at the predictor and corrector steps with time step $k / 2$ in an explicit MacCormack scheme we get

$$
\left.\left.\left.\left.\phi_{i j}^{\bar{*}}=\phi_{i j}^{n}+\frac{k}{2} \phi_{t}\right)_{i j}^{n}+\frac{k^{2}}{8} \phi_{2 t}\right)_{i j}^{n}+O\left(k^{3}\right), \phi_{i j}^{\bar{*}}=\phi_{i j}^{n}+\frac{k}{2} \phi_{t}\right)_{i j}^{\bar{*}}+\frac{k^{2}}{8} \phi_{2 t}\right)_{i j}^{\bar{*}}+O\left(k^{3}\right) .
$$

Using the definition of the operator $\mathrm{L}_{y}(\mathrm{k} / 2)$, we can consider the equation

$$
\phi_{t}+\mu \phi_{y}-a \phi_{y y}=f(\phi) \text {, which can be rewritten as } \phi_{t}=-\mu \phi_{y}+a \phi_{y y}+f(\phi) .
$$

From equation (2.3), the use of the chain rule provides

$$
\begin{aligned}
\phi_{2 t} & =\left(a \phi_{y y}-\mu \phi_{y}+f(\phi)\right)_{t} \\
& =a^{2} \phi_{4 y}-2 a \mu \phi_{3 y}+\mu^{2} \phi_{2 y}-\mu(f(\phi))_{y}+a(f(\phi))_{y y}+\left[-\mu \phi_{y}+a \phi_{y y}+f(\phi)\right] f^{\prime}(\phi) .
\end{aligned}
$$

This fact combined with equation (2.2) gives

$$
\begin{aligned}
\phi_{i j}^{\bar{*}}= & \phi_{i j}^{n}+\frac{k}{2}\left[a \phi_{y y}-\mu \phi_{y}+f(\phi)\right]_{i j}^{n}+\frac{k^{2}}{8}\left[a^{2} \phi_{4 y}-2 a \mu \phi_{3 y}+\mu^{2} \phi_{y y}-\mu(f(\phi))_{y}+a(f(\phi))_{y y}\right. \\
& \left.+\left[-\mu \phi_{y}+a \phi_{y y}+f(\phi)\right] f^{\prime}(\phi)\right]_{i j}^{n}+O\left(k^{3}\right) \\
\phi_{i j}^{\bar{*}}= & \phi_{i j}^{n}+\frac{k}{2}\left[a \phi_{y y}-\mu \phi_{y}+f(\phi)\right]_{i j}^{\bar{*}}+\frac{k^{2}}{8}\left[a^{2} \phi_{4 y}-2 a \mu \phi_{3 y}+\mu^{2} \phi_{y y}-\mu(f(\phi))_{y}+a(f(\phi))_{y y}\right. \\
& \left.+\left[-\mu \phi_{y}+a \phi_{y y}+f(\phi)\right] f^{\prime}(\phi)\right]_{i j}^{*}+O\left(k^{3}\right) .
\end{aligned}
$$

Expanding the Taylor series about $\left(x_{i}, y_{j}, t^{n}\right)$ and $\left(x_{i}, y_{j}, t^{\bar{*}}\right)$ with grid spacing $h$ using both forward and backward difference representations, it is not hard to see that

$$
\begin{aligned}
& \phi_{y, i j}^{n}=\delta_{y} \phi_{i, j+\frac{1}{2}}^{n}+O(h), \quad \phi_{y y, i j}^{n}=\delta_{y}^{2} \phi_{i j}^{n}+O\left(h^{2}\right), \quad \phi_{3 y, i j}^{n}=\delta_{y}^{2}\left(\delta_{y} \phi_{i, j+\frac{1}{2}}^{n}\right)+O(h), \\
& \phi_{4 y, i j}^{n}=\delta_{y}^{4} \phi_{i j}^{n}+O\left(h^{2}\right), \quad(f(\phi))_{y, i j}^{n}=\delta_{y}(f(\phi))_{i, j+\frac{1}{2}}^{n}+O(h), \quad(f(\phi))_{y y, i j}^{n}=\delta_{y}^{2}(f(\phi))_{i j}^{n}+O\left(h^{2}\right), \\
& \phi_{y, i j}^{\bar{*}}=\delta_{y} \phi_{i, j-\frac{1}{2}}^{\bar{*}}+\mathrm{O}(\mathrm{h}), \quad \phi_{y y, i j}^{\bar{*}}=\delta_{y}^{2} \phi_{i j}^{\bar{*}}+\mathrm{O}\left(\mathrm{h}^{2}\right), \quad \phi_{3 y, i j}^{\bar{*}}=\delta_{y}^{2}\left(\delta_{y} \phi_{i, j-\frac{1}{2}}^{\bar{*}}\right)+\mathrm{O}(\mathrm{h}), \\
& \phi_{4 y, i j}^{\bar{*}}=\delta_{y}^{4} \phi_{i j}^{\bar{*}}+O\left(h^{2}\right), \quad(f(\phi))_{y, i j}^{\bar{*}}=\delta_{y}(f(\phi))_{i, j-\frac{1}{2}}^{\bar{*}}+O(h), \quad(f(\phi))_{y y, i j}^{\bar{*}}=\delta_{y}^{2}(f(\phi))_{i j}^{\bar{*}}+O\left(h^{2}\right) .
\end{aligned}
$$

In relation (2.5), the operators $\delta_{y} w_{i j}^{l}$ and $\delta_{y}^{2} w_{i j}^{l}$ are defined by equation (2.1). Combining equations (2.4) and (2.5), straightforward computations yield

$$
\phi_{i j}^{\bar{F}}=\phi_{i j}^{n}+\frac{k}{2}\left[a \delta_{y}^{2} \phi_{i j}^{n}-\mu \delta_{y} \phi_{i, j+\frac{1}{2}}^{n}+f\left(\phi_{i j}^{n}\right)\right]+k^{2} \rho_{i j}^{n}+O\left(k^{3}+k h\right),
$$


and

$$
\phi_{i j}^{\bar{*}}=\phi_{i j}^{n}+\frac{k}{2}\left[a \delta_{y}^{2} \phi_{i j}^{\bar{*}}-\mu \delta_{y} \phi_{i, j-\frac{1}{2}}^{\bar{*}}+f\left(\phi_{i j}^{\bar{*}}\right)\right]+k^{2} \rho_{i j}^{\bar{*}}+O\left(k^{3}+k h\right),
$$

where

$$
\begin{aligned}
\rho_{i j}^{n}= & \frac{1}{8}\left\{a^{2} \delta_{y}^{2}\left(\delta_{y}^{2} \phi_{i j}^{n}\right)-2 a \mu \delta_{y}^{2}\left(\delta_{y} \phi_{i, j+\frac{1}{2}}^{n}\right)+\mu^{2} \delta_{y}^{2} \phi_{i j}^{n}-\mu \delta_{y}\left(f\left(\phi_{i, j+\frac{1}{2}}^{n}\right)\right)+a \delta_{y}^{2}\left(f\left(\phi_{i j}^{n}\right)\right)\right. \\
& \left.+\left[-\mu \delta_{y} \phi_{i, j+\frac{1}{2}}^{n}+a \delta_{y}^{2} \phi_{i j}^{n}+f\left(\phi_{i j}^{n}\right)\right] f^{\prime}\left(\phi_{i j}^{n}\right)\right\}, \\
\rho_{i j}^{\bar{*}}= & \frac{1}{8}\left\{a^{2} \delta_{y}^{2}\left(\delta_{y}^{2} \phi_{i j}^{\bar{*}}\right)-2 a \mu \delta_{y}^{2}\left(\delta_{y} \phi_{i, j-\frac{1}{2}}^{\bar{*}}\right)+\mu^{2} \delta_{y}^{2} \phi_{i j}^{\bar{*}}-\mu \delta_{y}\left(f\left(\phi_{i, j-\frac{1}{2}}^{\bar{*}}\right)\right)+a \delta_{y}^{2}\left(f\left(\phi_{i j}^{\bar{*}}\right)\right)\right. \\
& \left.+\left[-\mu \delta_{y} \phi_{i, j-\frac{1}{2}}^{\bar{*}}+a \delta_{y}^{2} \phi_{i j}^{\bar{*}}+f\left(\phi_{i j}^{\bar{*}}\right)\right] f^{\prime}\left(\phi_{i j}^{\bar{*}}\right)\right\} .
\end{aligned}
$$

Now, we should find a simple expression of the terms $f\left(\phi_{i j}^{\bar{*}}\right), \delta_{y} \phi_{i, j-\frac{1}{2}}^{\bar{*}}$, and $\delta_{y}^{2} \phi_{i j}^{\bar{*}}$. The application of the Taylor series expansion about $\left(x_{i}, y_{j}, t^{n}\right)$ with time step $\frac{k}{2}$ using forward difference representation results in

$$
f\left(\phi_{i j}^{*}\right)=f\left(\phi_{i j}^{n}\right)+\frac{k}{2}[f(\phi)]_{t, i j}^{n}+O\left(k^{2}\right)=f\left(\phi_{i j}^{n}\right)+\frac{k}{2} \phi_{t, i j}^{n} f^{\prime}\left(\phi_{i j}^{n}\right)+O\left(k^{2}\right) .
$$

But, it comes from equations (2.3) and (2.5) that

$$
\phi_{t, i j}^{n}=-\mu \phi_{y, i j}^{n}+a \phi_{y y, i j}^{n}+f\left(\phi_{i j}^{n}\right)=-\mu \delta_{y} \phi_{i, j+\frac{1}{2}}^{n}+a \delta_{y}^{2} \phi_{i j}^{n}+f\left(\phi_{i j}^{n}\right)+O(h) .
$$

A combination of equations (2.9) and (2.10) provides

$$
f\left(\phi_{i j}^{*}\right)=f\left(\phi_{i j}^{n}\right)+\frac{k}{2}\left[-\mu \delta_{y} \phi_{i, j+\frac{1}{2}}^{n}+a \delta_{y}^{2} \phi_{i j}^{n}+f\left(\phi_{i j}^{n}\right)\right] f^{\prime}\left(\phi_{i j}^{n}\right)+O\left(k^{2}+k h\right) .
$$

In addition, utilizing equation (2.6), it is not hard to see that

$$
\delta_{y} \phi_{i, j-\frac{1}{2}}^{\bar{*}}=\delta_{y} \phi_{i, j-\frac{1}{2}}^{n}+\frac{k}{2}\left[a \delta_{y}^{2}\left(\delta_{y} \phi_{i, j-\frac{1}{2}}^{n}\right)-\mu \delta_{y}^{2} \phi_{i j}^{n}+\delta_{y} f\left(\phi_{i, j-\frac{1}{2}}^{n}\right)\right]+O\left(k^{2}+k h\right),
$$

and

$$
\delta_{y}^{2} \phi_{i j}^{\bar{*}}=\delta_{y}^{2} \phi_{i j}^{n}+\frac{k}{2}\left[a \delta_{y}^{4} \phi_{i j}^{n}-\mu \delta_{y}^{2}\left(\delta_{y} \phi_{i, j+\frac{1}{2}}^{n}\right)+\delta_{y}^{2}\left(f\left(\phi_{i j}^{n}\right)\right]+O\left(k^{2}+k h\right),\right.
$$

Substituting equations (2.11)-(2.13) into (2.7), straightforward computations result in

$$
\phi_{i j}^{\bar{*}}=\phi_{i j}^{n}+\frac{k}{2}\left(a \delta_{y}^{2} \phi_{i j}^{n}-\mu \delta_{y} \phi_{i, j-\frac{1}{2}}^{n}+f\left(\phi_{i j}^{n}\right)\right)+k^{2}\left(\widehat{\rho}_{i j}^{n}+\rho_{i j}^{\bar{*}}\right)+O\left(k^{3}+k h\right),
$$

where

$$
\begin{aligned}
\widehat{\rho}_{i j}^{n}= & \frac{1}{4}\left\{a^{2} \delta_{y}^{4} \phi_{i j}^{n}-2 a \mu \delta_{y}^{2}\left(\delta^{y} \phi_{i j}^{n}\right)+\mu^{2} \delta_{y}^{2} \phi_{i j}^{n}-\mu \delta_{y}\left(f\left(\phi_{i, j-\frac{1}{2}}^{n}\right)\right)+a \delta_{y}^{2} f\left(\phi_{i j}^{n}\right)\right. \\
& \left.+\left[-\mu \delta_{y} \phi_{i, j+\frac{1}{2}}^{n}+a \delta_{y}^{2} \phi_{i j}^{n}+f\left(\phi_{i j}^{n}\right)\right] f^{\prime}\left(\phi_{i j}^{n}\right)\right\} .
\end{aligned}
$$

Plugging equations (2.6) and (2.14), simple calculations give

$$
\frac{1}{2}\left(\phi_{i j}^{\bar{*}}+\phi_{i j}^{\bar{*}}\right)=\phi_{i j}^{n}+\frac{k}{2}\left(a \delta_{y}^{2} \phi_{i j}^{n}-\mu \delta^{y} \phi_{i j}^{n}+f\left(\phi_{i j}^{n}\right)\right)+\frac{k^{2}}{2}\left(\rho_{i j}^{n}+\widehat{\rho}_{i j}^{n}+\rho_{i j}^{\bar{*}}\right)+O\left(k^{3}+k h^{2}\right),
$$

where $\delta^{y} \phi_{i j}^{n}=\frac{\delta_{y} \phi_{i, j+\frac{1}{2}}^{n}+\delta_{y} \phi_{i, j-\frac{1}{2}}^{n}}{2}$, and $\rho_{i j}^{\alpha}$ and $\widehat{\rho}_{i j}^{n}$ are defined by relations (2.8) and (2.15), respectively. The second order infinitesimal term $\mathrm{O}\left(\mathrm{h}^{2}\right)$ in equation (2.16) comes from the Taylor expansion $\left.\phi_{y}\right)_{i j}^{n}=$ $\delta^{y} \phi_{i j}^{n}+O\left(h^{2}\right)$. 
In similar way, to define the operator $L_{x}(k)$, we should consider the following equation

$$
\phi_{\mathrm{t}}+\mu \phi_{\mathrm{x}}-\mathrm{a} \phi_{\mathrm{xx}}=0 \text {, which is equivalent to } \phi_{\mathrm{t}}=-\mu \phi_{\mathrm{x}}+\mathrm{a} \phi_{\mathrm{x} x} .
$$

Utilizing equation (2.17), it is not difficult to observe that

$$
\phi_{2 t}=\left(-\mu \phi_{x}+a \phi_{x x}\right)_{t}=\mu^{2} \phi_{2 x}-2 a \mu \phi_{3 x}+a^{2} \phi_{4 x} .
$$

Expanding the Taylor series about $\left(x_{i}, y_{i}, t^{*}\right)$ (where $t^{*} \in\left(t^{n}, t^{n+1}\right)$, is the starting time at the next step in a time-split MacCormack scheme) with grid spacing $h$ using both forward and backward difference representations we get

$$
\begin{aligned}
& \phi_{x, i j}^{*}=\delta_{x} \phi_{i+\frac{1}{2}, j}^{*}+O(h), \quad \phi_{x x, i j}^{*}=\delta_{x}^{2} \phi_{i j}^{*}+O\left(h^{2}\right), \quad \phi_{3 x, i j}^{*}=\delta_{x}^{2}\left(\delta_{x} \phi_{i+\frac{1}{2}, j}^{*}\right)+O(h), \\
& \phi_{4 x, i j}^{*}=\delta_{x}^{4} \phi_{i j}^{*}+\mathrm{O}\left(h^{2}\right), \quad \phi_{x, i j}^{* *}=\delta_{x} \phi_{i-\frac{1}{2}, j}^{* *}+\mathrm{O}(\mathrm{h}), \quad \phi_{x x, i j}^{* *}=\delta_{x}^{2} \phi_{i j}^{* *}+\mathrm{O}\left(h^{2}\right), \\
& \phi_{3 x, i j}^{\overline{* *}}=\delta_{x}^{2}\left(\delta_{x} \phi_{i-\frac{1}{2}, j}^{\overline{* *}}\right)+\mathrm{O}(\mathrm{h}), \quad \phi_{4 x, i j}^{* *}=\delta_{x}^{4} \phi_{i j}^{* *}+\mathrm{O}\left(\mathrm{h}^{2}\right),
\end{aligned}
$$

where $\delta_{x} \phi_{i \pm \frac{1}{2}, j}^{l}$ and $\delta_{x}^{2} \phi_{i j}^{l}$ are defined by equation (2.1). Furthermore, the application of the Taylor series at the predictor and corrector steps about $\left(x_{i}, y_{j}, t^{*}\right)$ with time step $k$ using forward difference representation provides

$$
\left.\left.\left.\left.\phi_{i j}^{\overline{* *}}=\phi_{i j}^{*}+k \phi_{t}\right)_{i j}^{*}+\frac{k^{2}}{2} \phi_{2 t}\right)_{i j}^{*}+O\left(k^{3}\right), \phi_{i j}^{\overline{* *}}=\phi_{i j}^{*}+k \phi_{t}\right)_{i j}^{\overline{* *}}+\frac{k^{2}}{2} \phi_{2 t}\right)_{i j}^{\overline{* *}}+O\left(k^{3}\right) .
$$

Combining equations (2.17), (2.18), (2.19), and (2.20), direct computations result in

$$
\phi_{i j}^{* *}=\phi_{i j}^{*}+k\left[-\mu \delta_{x} \phi_{i+\frac{1}{2}, j}^{*}+a \delta_{x}^{2} \phi_{i j}^{*}\right]+k^{2} \gamma_{i j}^{*}+O\left(k^{3}+k h\right),
$$

and

$$
\phi_{i j}^{\overline{* *}}=\phi_{i j}^{*}+k\left[-\mu \delta_{x} \phi_{i-\frac{1}{2}, j}^{\overline{* *}}+a \delta_{x}^{2} \phi_{i j}^{\overline{* *}}\right]+k^{2} \gamma_{i j}^{\overline{* *}}+\mathrm{O}\left(k^{3}+k h\right),
$$

where

$$
\gamma_{i j}^{*}=\frac{1}{2}\left[a^{2} \delta_{x}^{2}\left(\delta_{x}^{2} \phi_{i j}^{*}\right)-2 a \mu \delta_{x}^{2}\left(\delta_{x} \phi_{i+\frac{1}{2}, j}^{*}\right)+\mu^{2} \delta_{x}^{2} \phi_{i j}^{*}\right], \gamma_{i j}^{* *}=\frac{1}{2}\left[a^{2} \delta_{x}^{2}\left(\delta_{x}^{2} \phi_{i j}^{* *}\right)-2 a \mu \delta_{x}^{2}\left(\delta_{x} \phi_{i-\frac{1}{2}, j}^{\overline{* *}}\right)+\mu^{2} \delta_{x}^{2} \phi_{i j}^{* *}\right] .
$$

We should find an explicit expression of terms $\delta_{x}^{2} \phi_{i j}^{\overline{* *}}$ and $\delta_{x} \phi_{i-\frac{1}{2}, j}^{\overline{* *}}$. Using equation (2.21), simple calculations yield

$$
\delta_{x}^{2} \phi_{i j}^{* *}=\delta_{x}^{2} \phi_{i j}^{*}+k\left[-\mu \delta_{x}^{2}\left(\delta_{x} \phi_{i+\frac{1}{2}, j}^{*}\right)+a \delta_{x}^{4} \phi_{i j}^{*}\right]+O\left(k^{2}+k h\right),
$$

and

$$
\delta_{x} \phi_{i-\frac{1}{2}, j}^{* *}=\delta_{x} \phi_{i-\frac{1}{2}, j}^{*}+k\left[-\mu \delta_{x}^{2} \phi_{i j}^{*}+a \delta_{x}^{2}\left(\delta_{x} \phi_{i-\frac{1}{2}, j}^{*}\right)\right]+\mathrm{O}\left(k^{2}+k h\right) .
$$

This fact, together with relation (2.22) yield

$$
\phi_{i j}^{\overline{* *}}=\phi_{i j}^{*}+k\left(a \delta_{x}^{2} \phi_{i j}^{*}-\mu \delta_{x} \phi_{i-\frac{1}{2}, j}^{*}\right)+k^{2}\left(\widehat{\gamma}_{i j}^{*}+\gamma_{i j}^{* *}\right)+O\left(k^{3}+k h\right),
$$

where

$$
\widehat{\gamma}_{i j}^{*}=\mu^{2} \delta_{x}^{2} \phi_{i j}^{*}-2 a \mu \delta_{x}^{2}\left(\delta^{x} \phi_{i j}^{*}\right)+a^{2} \delta_{x}^{4} \phi_{i j}^{*} .
$$

A combination of equations (2.21) and (2.23) results in

$$
\frac{1}{2}\left(\phi_{i j}^{\overline{* *}}+\phi_{i j}^{\overline{* *}}\right)=\phi_{i j}^{*}+k\left(-\mu \delta^{x} \phi_{i j}^{*}+a \delta_{x}^{2} \phi_{i j}^{*}\right)+\frac{k^{2}}{2}\left(\gamma_{i j}^{*}+\widehat{\gamma}_{i j}^{*}+\gamma_{i j}^{* *}\right)+O\left(k^{3}+k h^{2}\right),
$$


where the second order error term $\mathrm{O}\left(\mathrm{h}^{2}\right)$ comes from the approximation

$$
\phi_{x, i j}^{*}=\delta^{x} \phi_{i j}^{*}+O\left(h^{2}\right)=\frac{1}{2}\left(\delta_{x} \phi_{i+\frac{1}{2}, j}^{*}+\delta_{x} \phi_{i-\frac{1}{2}, j}^{*}\right)+O\left(h^{2}\right) .
$$

Similarly, starting with the one-dimensional equation: $\phi_{t}+\mu \phi_{y}-a \phi_{y y}=f(\phi)$, expanding the Taylor series about $\left(x_{i}, y_{j}, t^{* *}\right)$ (where $t^{* *}$ is the time used at the last step in a time-split MacCormack technique) at both predictor and corrector steps with time step $k / 2$ and mesh size $h$, using forward and backward difference representations, it is not difficult to show that

$$
\frac{1}{2}\left(\phi_{i j}^{\overline{n+1}}+\phi_{i j}^{\overline{n+1}}\right)=\phi_{i j}^{* *}+\frac{k}{2}\left(-\mu \delta^{y} \phi_{i j}^{* *}+a \delta_{y}^{2} \phi_{i j}^{* *}+f\left(\phi_{i j}^{* *}\right)\right)+\frac{k^{2}}{2}\left(\theta_{i j}^{* *}+\widehat{\theta}_{i j}^{* *}+\theta_{i j}^{\overline{n+1}}\right)+O\left(k^{3}+k h^{2}\right),
$$

where

$$
\begin{aligned}
\theta_{i j}^{\alpha}= & \frac{1}{8}\left\{a^{2} \delta_{y}^{2}\left(\delta_{y}^{2} \phi_{i j}^{\alpha}\right)-2 a \mu \delta_{y}^{2}\left(\delta_{y} \phi_{i, j \pm \frac{1}{2}}^{\alpha}\right)+\mu^{2} \delta_{y}^{2} \phi_{i j}^{\alpha}-\mu \delta_{y}\left(f\left(\phi_{i, j \pm \frac{1}{2}}^{\alpha}\right)\right)+a \delta_{y}^{2}\left(f\left(\phi_{i j}^{\alpha}\right)\right)\right. \\
& \left.+\left[-\mu \delta_{y} \phi_{i, j \pm \frac{1}{2}}^{\alpha}+a \delta_{y}^{2} \phi_{i j}^{\alpha}+f\left(\phi_{i j}^{\alpha}\right)\right] f^{\prime}\left(\phi_{i j}^{\alpha}\right)\right\}
\end{aligned}
$$

where we set $\alpha=* *, \overline{\mathrm{n}+1}$ and

$$
\begin{aligned}
\widehat{\theta}_{i j}^{* *}= & \frac{1}{4}\left\{a^{2} \delta_{y}^{4} \phi_{i j}^{* *}-2 a \mu \delta_{y}^{2}\left(\delta^{y} \phi_{i j}^{* *}\right)+\mu^{2} \delta_{y}^{2} \phi_{i j}^{* *}-\mu \delta_{y}\left(f\left(\phi_{i, j-\frac{1}{2}}^{* *}\right)\right)+a \delta_{y}^{2} f\left(\phi_{i j}^{* *}\right)\right. \\
& \left.+\left[-\mu \delta_{y} \phi_{i, j+\frac{1}{2}}^{* *}+a \delta_{y}^{2} \phi_{i j}^{* *}+f\left(\phi_{i j}^{* *}\right)\right] f^{\prime}\left(\phi_{i j}^{* *}\right)\right\},
\end{aligned}
$$

with $\delta^{y} \phi_{i j}^{\alpha}=\frac{\delta_{y} \phi_{i, j+\frac{1}{2}}^{\alpha}+\delta_{y} \phi_{i, j-\frac{1}{2}}^{\alpha}}{2}$.

In order to provide a full description of a three-level explicit time-split MacCormack method for solving the two-dimensional nonlinear time-dependent convection-diffusion-reaction equation (1.1) with initial and boundary conditions (1.2) and (1.3), respectively, we should follow the ideas developed in $[21,27,32]$. We must neglect the second order terms together with the error term $\mathrm{O}\left(\mathrm{k}^{3}+\mathrm{kh}^{2}\right)$ in equations (2.16), (2.24), and (2.25). Furthermore, the terms $\phi_{i j}^{*}, \phi_{i j}^{* *}$, and $\phi_{i j}^{n+1}$ are defined as the average of predicted and corrected values,

$$
\phi_{i j}^{*}=\frac{\phi_{i j}^{*}+\phi_{i j}^{\bar{*}}}{2}, \phi_{i j}^{* *}=\frac{\phi_{i j}^{\overline{* *}}+\phi_{i j}^{\overline{* *}}}{2}, \quad \text { and } \quad \phi_{i j}^{n+1}=\frac{\phi_{i j}^{\overline{n+1}}+\phi_{i j}^{\overline{n+1}}}{2} \text {. }
$$

By definition, equations

$$
\phi_{i j}^{*}=L_{y}(k / 2) \phi_{i j}^{n}, \quad \phi_{i j}^{* *}=L_{x}(k) \phi_{i j}^{*}, \quad \text { and } \quad \phi_{i j}^{n+1}=L_{y}(k / 2) \phi_{i j}^{* *},
$$

are equivalent to

$$
\begin{aligned}
\phi_{i j}^{*} & =\phi_{i j}^{n}+\frac{k}{2}\left(-\mu \delta^{y} \phi_{i j}^{n}+a \delta_{y}^{2} \phi_{i j}^{n}+f\left(\phi_{i j}^{n}\right)\right), \\
\phi_{i j}^{* *} & =\phi_{i j}^{*}+k\left(-\mu \delta^{x} \phi_{i j}^{*}+a \delta_{x}^{2} \phi_{i j}^{*}\right), \\
\phi_{i j}^{n+1} & =\phi_{i j}^{* *}+\frac{k}{2}\left(-\mu \delta^{y} \phi_{i j}^{* *}+a \delta_{y}^{2} \phi_{i j}^{* *}+f\left(\phi_{i j}^{* *}\right)\right) .
\end{aligned}
$$

From the definition of the operators $L_{y}(k / 2)$ and $L_{x}(k)$, it is not hard to observe that the operator $\mathrm{L}_{y}(\mathrm{k} / 2) \mathrm{L}_{x}(\mathrm{k}) \mathrm{L}_{y}(\mathrm{k} / 2)$ is symmetric. This fact, together with relations (2.16), (2.24), and (2.25) suggest that the new method is a three-level approach, an explicit predictor-corrector scheme, second order accurate in time and fourth order convergent in space. This theoretical result is confirmed by a wide set of numerical examples (see Section 5). Furthermore, it comes from the definition of the linear operators 
$" \delta^{x \prime}, " \delta^{y},, " \delta_{x}^{2} "$ and $" \delta_{y}^{2}$ " given in relation (2.1) that equation (2.28) can be rewritten as follows. For $\mathrm{n}=0,1, \ldots, \mathrm{N}-1$;

$$
\begin{aligned}
\phi_{i j}^{*} & =\phi_{i j}^{n}+\frac{k}{2}\left[\frac{a}{h^{2}}\left(\phi_{i, j+1}^{n}-2 \phi_{i j}^{n}+\phi_{i, j-1}^{n}\right)-\frac{\mu}{2 h}\left(\phi_{i, j+1}^{n}-\phi_{i, j-1}^{n}\right)+f\left(\phi_{i j}^{n}\right)\right], i=0,1, \ldots, M ; j=1, \ldots, M-1, \\
\phi_{i j}^{* *} & =\phi_{i j}^{*}+k\left[\frac{a}{h^{2}}\left(\phi_{i+1, j}^{*}-2 \phi_{i j}^{*}+\phi_{i-1, j}^{*}\right)-\frac{\mu}{2 h}\left(\phi_{i+1, j}^{*}-\phi_{i-1, j}^{*}\right)\right], i=1,2, \ldots, M-1 ; j=0,1, \ldots, M, \\
\phi_{i j}^{n+1} & =\phi_{i j}^{* *}+\frac{k}{2}\left[\frac{a}{h^{2}}\left(\phi_{i, j+1}^{* *}-2 \phi_{i j}^{* *}+\phi_{i, j-1}^{* *}\right)-\frac{\mu}{2 h}\left(\phi_{i, j+1}^{* *}-\phi_{i, j-1}^{* *}\right)+f\left(\phi_{i j}^{* *}\right)\right], i=0,1, \ldots, M ; j=1, \ldots, M-1,
\end{aligned}
$$

subject to the initial and boundary conditions. For $i, j=0,1, \ldots, M$,

$$
\begin{aligned}
& \phi_{i j}^{0}=\phi_{0}\left(x_{i}, y_{j}\right), \quad \phi_{i 0}^{n}=\varphi_{i 0}^{n}, \quad \phi_{i M}^{n}=\varphi_{i M}^{n}, \quad \phi_{0 j}^{n}=\varphi_{0 j}^{n}, \quad \phi_{M j}^{n}=\varphi_{M j}^{n}, \quad \phi_{0 j}^{*}=\varphi_{0 j}^{n+1}, \\
& \phi_{M j}^{*}=\varphi_{M j}^{n+1}, \quad \phi_{j 0}^{*}=\varphi_{j 0}^{n+1}, \quad \phi_{j M}^{*}=\varphi_{j M}^{n+1}, \quad \phi_{0 j}^{* *}=\varphi_{0 j}^{n+1}, \quad \phi_{M j}^{* *}=\varphi_{M j}^{n+1}, \quad \phi_{j 0}^{* *}=\varphi_{j 0}^{n+1}, \\
& \phi_{j M}^{* *}=\varphi_{j M}^{n+1}, \quad \phi_{i 0}^{N}=\varphi_{i 0}^{N}, \quad \phi_{i M}^{N}=\varphi_{i M}^{N}, \quad \phi_{0 j}^{N}=\varphi_{0 j}^{N}, \quad \phi_{M j}^{N}=\varphi_{M j}^{N} .
\end{aligned}
$$

Equations (2.29)-(2.32) represent a detailed description of a three-level explicit time-split MacCormack method applied to nonlinear convection-diffusion-reaction equations (1.1)-(1.3).

In the following, we analyze the stability, the error estimates and the convergence rate of the numerical scheme (2.29)-(2.32), under the time step requirement

$$
\max \left\{\frac{2 a k}{h^{2}}, \frac{|\mu| k}{h}\right\} \leqslant 1
$$

where $a>0$ and $\mu$ are physical parameters given in equation (1.1). We assume that the analytical solution $\bar{\phi} \in \mathrm{L}^{\infty}\left(0, \mathrm{~T} ; \mathrm{L}^{2}(\Omega)\right) \cap \mathrm{H}^{1}\left(0, \mathrm{~T} ; \mathrm{H}^{3}(\Omega)\right) \cap \mathrm{H}^{2}\left(0, \mathrm{~T} ; \mathrm{H}^{1}(\Omega)\right) \cap \mathrm{H}^{2}\left(0, \mathrm{~T} ; \mathrm{L}^{2}(\Omega)\right) \cap \mathrm{L}^{2}\left(0, \mathrm{~T} ; \mathrm{H}^{4}(\Omega)\right)$, that is, there is a positive constant $\widetilde{C}$, that does not depend neither on the time step $k$ nor the mesh size $h$, so that

$$
\||\bar{\phi}|\|_{\mathrm{L}^{\infty}\left(0, \mathrm{~T} ; \mathrm{L}^{2}(\Omega)\right)}+\left\|\bar{\phi}\left|\left\|_{\mathrm{H}^{1}\left(0, \mathrm{~T} ; \mathrm{H}^{3}(\Omega)\right)}+\right\|\right| \bar{\phi} \mid\right\|_{\mathrm{H}^{2}\left(0, \mathrm{~T} ; \mathrm{H}^{1}(\Omega)\right)}+\|\bar{\phi}\|_{\mathrm{H}^{2}\left(0, \mathrm{~T} ; \mathrm{L}^{2}(\Omega)\right)}+\|\bar{\phi}\| \|_{\mathrm{L}^{2}\left(0, \mathrm{~T} ; \mathrm{H}^{4}(\Omega)\right)} \leqslant \widetilde{\mathrm{C}}
$$

To end this section, we state the following result (Lemma 2.1) which plays a crucial role in the proof of the stability analysis of the numerical scheme (Theorem 3.1).

Lemma 2.1. Let $\phi_{i j}^{n}=\phi\left(x_{i}, y_{j}, t^{n}\right)$ be the numerical solution provided by the scheme (2.29)-(2.32), $\bar{\phi}_{i j}^{n}=$ $\bar{\phi}\left(x_{i}, y_{j}, t^{n}\right)$ be the exact one and let $e_{i j}^{n}=\phi_{i j}^{n}-\bar{\phi}_{i j}^{n}$ be the error. We recall that $\bar{\phi}_{i j}^{*}=\frac{\bar{\phi}_{i j}^{*}+\bar{\phi}_{i j}^{\bar{*}}}{2}$ and $\bar{\phi}_{i j}^{* *}=\frac{\bar{\phi}_{i j}^{* *}+\bar{\phi}_{i j}^{\overline{* *}}}{2}$, satisfy relations (2.16) and (2.24), respectively. $\phi_{i j}^{*}$ and $\phi_{i j}^{* *}$ are given by equations (2.29) and (2.30), respectively. Then, it holds

$$
\begin{aligned}
& a<\delta_{x}^{2} e_{i j}^{n}, e_{i j}^{n}>_{x}=h^{2} \sum_{j, i=1}^{M-1} \frac{a}{h^{2}}\left(e_{i+1, j}^{n}-2 e_{i j}^{n}+e_{i-1, j}^{n}\right) e_{i j}^{n}=-a\left\|\delta_{x} e^{n}\right\|_{L^{2}(\Omega)}^{2}, \\
& a<\delta_{y}^{2} e_{i j}^{n}, e_{i j}^{n}>_{y}=h^{2} \sum_{j, i=1}^{M-1} \frac{a}{h^{2}}\left(e_{i, j+1}^{n}-2 e_{i j}^{n}+e_{i, j-1}^{n}\right) e_{i j}^{n}=-a\left\|\delta_{y} e^{n}\right\|_{L^{2}(\Omega)}^{2},
\end{aligned}
$$

and

$$
\sum_{j, i=1}^{M-1}\left(e_{i, j+1}^{n}-e_{i, j-1}^{n}\right) e_{i j}^{n}=0
$$

Proof. Equations (2.35) and (2.36) are proved in [27].

Now, let prove equation (2.37). Firstly, it is easy to see that

$$
\left(e_{i, j+1}^{n}-e_{i, j-1}^{n}\right) e_{i j}^{n}=e_{i, j+1}^{n} e_{i j}^{n}-e_{i j}^{n} e_{i, j-1}^{n} .
$$


Summing this up from $i, j=1,2, \ldots, M-1$, to get

$$
\sum_{i, j=1}^{M-1}\left(e_{i, j+1}^{n}-e_{i, j-1}^{n}\right) e_{i j}^{n}=\sum_{i, j=1}^{M-1}\left(e_{i, j+1}^{n} e_{i j}^{n}-e_{i j}^{n} e_{i, j-1}^{n}\right)=\sum_{i=1}^{M-1}\left(e_{i M}^{n} e_{i, M-1}^{n}-e_{i 1}^{n} e_{i 0}^{n}\right) .
$$

But, it comes from the boundary condition (2.32) that $e_{i M}^{n}=e_{i 0}^{n}=0$. Using this fact, equation (2.38) becomes

$$
\sum_{i, j=1}^{M-1}\left(e_{i, j+1}^{n}-e_{i, j-1}^{n}\right) e_{i j}^{n}=0
$$

With the above tools, we are ready to analyze the stability of the three-level time-split MacCormack method (2.29)-(2.32) applied to the initial-boundary value problem (1.1)-(1.3).

\section{Stability analysis of a three-level time-split MacCormack scheme}

This section considers the stability analysis of the numerical scheme (2.29)-(2.32) for solving the twodimensional nonlinear unsteady convection-diffusion-reaction equations (1.1)-(1.3).

Theorem 3.1. Consider $\phi$ to be the numerical solution obtained from the scheme (2.29)-(2.32). Under the time step restriction (2.33), the approximate solution $\phi$ satisfies

$$
\max _{0 \leqslant n \leqslant N}\left\|\phi^{n}\right\|_{L^{2}(\Omega)} \leqslant \widetilde{C}+\exp \left(\frac{7 C T}{2} g(C, \mu, a)(1+g(C, \mu, a))^{2} \sum_{l=0}^{5}(C k)^{l}\right),
$$

where $\mathrm{g}(\mathrm{C}, \mu, \mathrm{a})=1+\frac{\mu^{2}}{4 \mathrm{aC}}+\frac{\mathrm{C}}{8}+\frac{9}{4 \mathrm{C}}, \mathrm{C}$ is a positive constant independent of the time step $\mathrm{k}$ and the mesh size $\mathrm{h}$, $\mu$ and $\mathrm{a}>0$, are two physical parameters and $\widetilde{\mathrm{C}}$ is the constant given by equation (2.34).

Proof. Plugging equations (2.16), (2.27), and (2.29), straightforward computations result in

$$
e_{i j}^{*}=e_{i j}^{n}+\frac{k}{2}\left(a \delta_{y}^{2} e_{i j}^{n}-\mu \delta^{y} e_{i j}^{n}+f\left(\phi_{i j}^{n}\right)-f\left(\bar{\phi}_{i j}^{n}\right)\right)+\frac{k^{2}}{2}\left(\rho_{i j}^{n}+\widehat{\rho}_{i j}^{n}+\rho_{i j}^{\bar{*}}\right)+O\left(k^{3}+k h^{2}\right),
$$

where $\rho_{i j}^{\alpha}(\alpha=n, \bar{*})$ and $\widehat{\rho}_{i j}^{n}$ are given by (2.8) and (2.15), respectively. From the definition of the linear operator " $\delta_{y}^{2}$ ", equation (3.1) can be rewritten as

$$
\begin{aligned}
e_{i j}^{*}= & e_{i j}^{n}+\frac{k}{2}\left(\frac{a}{h^{2}}\left(e_{i, j+1}^{n}-2 e_{i j}^{n}+e_{i, j-1}^{n}\right)-\frac{\mu}{2 h}\left(e_{i, j+1}^{n}-e_{i, j-1}^{n}\right)+f\left(\phi_{i j}^{n}\right)-f\left(\bar{\phi}_{i j}^{n}\right)\right) \\
& +\frac{k^{2}}{2}\left(\rho_{i j}^{n}+\hat{\rho}_{i j}^{n}+\rho_{i j}^{\bar{*}}\right)+O\left(k^{3}+k h^{2}\right) .
\end{aligned}
$$

Since the formulas can become quite heavy, for the sake of readability, we must neglect the terms of higher order in time step $k$ and mesh grid $h$. Because the aim of this section is to analyze the stability of the numerical scheme, the truncation of the second order term and the infinitesimal term does not compromise the result. This fact, together with equation (3.2) provides

$$
e_{i j}^{*}=e_{i j}^{n}+\frac{k}{2}\left(\frac{a}{h^{2}}\left(e_{i, j+1}^{n}-2 e_{i j}^{n}+e_{i, j-1}^{n}\right)-\frac{\mu}{2 h}\left(e_{i, j+1}^{n}-e_{i, j-1}^{n}\right)+f\left(\phi_{i j}^{n}\right)-f\left(\bar{\phi}_{i j}^{n}\right)\right) .
$$

Taking the square, equation (3.3) yields

$$
\begin{aligned}
\left(e_{i j}^{*}\right)^{2}= & \left(e_{i j}^{n}\right)^{2}+k\left[\frac{a}{h^{2}}\left(e_{i, j+1}^{n}-2 e_{i j}^{n}+e_{i, j-1}^{n}\right)-\frac{\mu}{2 h}\left(e_{i, j+1}^{n}-e_{i, j-1}^{n}\right)+f\left(\phi_{i j}^{n}\right)-f\left(\bar{\phi}_{i j}^{n}\right)\right] e_{i j}^{n} \\
& +\frac{k^{2}}{4}\left(\frac{a}{h^{2}}\left(e_{i, j+1}^{n}-2 e_{i j}^{n}+e_{i, j-1}^{n}\right)-\frac{\mu}{2 h}\left(e_{i, j+1}^{n}-e_{i, j-1}^{n}\right)+f\left(\phi_{i j}^{n}\right)-f\left(\bar{\phi}_{i j}^{n}\right)\right)^{2} .
\end{aligned}
$$


Applying equality $(a-b)(a+b)=a^{2}-b^{2}$, together with inequalities $(a \pm b)^{2} \leqslant 2\left(a^{2}+b^{2}\right)$ and $(a \pm b \pm$ $c)^{2} \leqslant 3\left(a^{2}+b^{2}+c^{2}\right)$, for any $a, b, c \in \mathbb{R}$, direct calculations give

$$
\begin{aligned}
\left(e_{i, j+1}^{n}-2 e_{i j}^{n}+e_{i, j-1}^{n}\right)^{2} & \leqslant 2\left[\left(e_{i, j+1}^{n}-e_{i j}^{n}\right)^{2}+\left(e_{i, j-1}^{n}-e_{i j}^{n}\right)^{2}\right], \\
\left(e_{i, j+1}^{n}-e_{i, j-1}^{n}\right)^{2} & \leqslant 2\left[\left(e_{i, j+1}^{n}\right)^{2}+\left(e_{i, j-1}^{n}\right)^{2}\right], \\
\frac{2 a}{h^{2}}\left(e_{i, j+1}^{n}-2 e_{i j}^{n}+e_{i, j-1}^{n}\right)\left(f\left(\phi_{i j}^{n}\right)-f\left(\bar{\phi}_{i j}^{n}\right)\right) & \leqslant \frac{a}{h^{2}}\left(e_{i, j+1}^{n}-2 e_{i j}^{n}+e_{i, j-1}^{n}\right)^{2}+\frac{a}{h^{2}}\left(f\left(\phi_{i j}^{n}\right)-f\left(\bar{\phi}_{i j}^{n}\right)\right)^{2}, \\
\left(e_{i, j+1}^{n}-e_{i, j-1}^{n}\right)\left(e_{i, j+1}^{n}-2 e_{i j}^{n}+e_{i, j-1}^{n}\right)= & {\left[\left(e_{i, j+1}^{n}-e_{i j}^{n}\right)+\left(e_{i j}^{n}-e_{i, j-1}^{n}\right)\right] } \\
& \times\left[\left(e_{i, j+1}^{n}-e_{i j}^{n}\right)-\left(e_{i j}^{n}-e_{i, j-1}^{n}\right)\right] \\
& =\left(e_{i, j+1}^{n}-e_{i j}^{n}\right)^{2}-\left(e_{i j}^{n}-e_{i, j-1}^{n}\right)^{2}, \\
\frac{\mu}{h}\left(e_{i, j+1}^{n}-e_{i, j-1}^{n}\right)\left(f\left(\phi_{i j}^{n}\right)-f\left(\bar{\phi}_{i j}^{n}\right)\right) & \leqslant \frac{\mu^{2}}{4 h^{2}}\left(e_{i, j+1}^{n}-e_{i, j-1}^{n}\right)^{2}+\left(f\left(\phi_{i j}^{n}\right)-f\left(\bar{\phi}_{i j}^{n}\right)\right)^{2} .
\end{aligned}
$$

Since $f \in \mathcal{C}^{1}(\mathbb{R})$ is a Lipschitz function, there exists a positive constant $C$ independent of the time step $k$ and the grid spacing $h$ so that

$$
\left|f\left(\phi_{i j}^{n}\right)-f\left(\bar{\phi}_{i j}^{n}\right)\right| \leqslant C\left|e_{i j}^{n}\right| .
$$

Using estimate (3.10), it is not difficult to see that

$$
\left(f\left(\phi_{i j}^{n}\right)-f\left(\bar{\phi}_{i j}^{n}\right)\right) e_{i j}^{n} \leqslant C\left(e_{i j}^{n}\right)^{2} \text { and }\left(f\left(\phi_{i j}^{n}\right)-f\left(\bar{\phi}_{i j}^{n}\right)\right)^{2} \leqslant C^{2}\left(e_{i j}^{n}\right)^{2}
$$

From estimates (3.5)-(3.11), it holds

$$
\begin{aligned}
& {\left[\frac{a}{h^{2}}\left(e_{i, j+1}^{n}-2 e_{i j}^{n}+e_{i, j-1}^{n}\right)-\frac{\mu}{2 h}\left(e_{i, j+1}^{n}-e_{i, j-1}^{n}\right)+f\left(\phi_{i j}^{n}\right)-f\left(\bar{\phi}_{i j}^{n}\right)\right]^{2} } \\
&=\frac{\mu^{2}}{4 h^{2}}\left(e_{i, j+1}^{n}-e_{i, j-1}^{n}\right)^{2}+\frac{a^{2}}{h^{4}}\left(e_{i, j+1}^{n}-2 e_{i j}^{n}+e_{i, j-1}^{n}\right)^{2} \\
& \quad+\left(f\left(\phi_{i j}^{n}\right)-f\left(\bar{\phi}_{i j}^{n}\right)\right)^{2}-\frac{a \mu}{h^{3}}\left(e_{i, j+1}^{n}-e_{i, j-1}^{n}\right)\left(e_{i, j+1}^{n}-2 e_{i j}^{n}+e_{i, j-1}^{n}\right) \\
& \quad-\frac{\mu}{h}\left(e_{i, j+1}^{n}-e_{i, j-1}^{n}\right)\left(f\left(\phi_{i j}^{n}\right)-f\left(\bar{\phi}_{i j}^{n}\right)\right)+\frac{2 a}{h^{2}}\left(e_{i, j+1}^{n}-2 e_{i j}^{n}+e_{i, j-1}^{n}\right)\left(f\left(\phi_{i j}^{n}\right)-f\left(\bar{\phi}_{i j}^{n}\right)\right) \\
& \leqslant \frac{\mu^{2}}{2 h^{2}}\left(e_{i, j+1}^{n}-e_{i, j-1}^{n}\right)^{2}+\frac{2 a^{2}}{h^{2}}\left[\delta_{y}\left(e_{i, j+\frac{1}{2}}^{n}\right)^{2}+\delta_{y}\left(e_{i, j-\frac{1}{2}}^{n}\right)^{2}\right]+\left(2+\frac{a}{h^{2}}\right)\left(f\left(\phi_{i j}^{n}\right)-f\left(\bar{\phi}_{i j}^{n}\right)\right)^{2} \\
&+\frac{a}{h^{2}}\left(e_{i, j+1}-2 e_{i j}^{n}+e_{i, j-1}^{n}\right)^{2}-\frac{a \mu}{h}\left[\delta_{y}\left(e_{i, j+\frac{1}{2}}^{n}\right)^{2}-\delta_{y}\left(e_{i, j-\frac{1}{2}}^{n}\right)^{2}\right] \\
& \leqslant \frac{\mu^{2}}{h^{2}}\left[\left(e_{i, j+1}^{n}\right)^{2}+\left(e_{i, j-1}^{n}\right)^{2}\right]+\frac{2 a^{2}}{h^{2}}\left[\delta_{y}\left(e_{i, j+\frac{1}{2}}^{n}\right)^{2}+\delta_{y}\left(e_{i, j-\frac{1}{2}}^{n}\right)^{2}\right]+C^{2}\left(2+\frac{a}{h^{2}}\right)\left(e_{i j}^{n}\right)^{2} \\
&+\frac{3 a}{h^{2}}\left[\left(e_{i, j+1}^{n}\right)^{2}+4\left(e_{i j}^{n}\right)^{2}+\left(e_{i, j-1}^{n}\right)^{2}\right]+\frac{a \mu}{h}\left[\delta_{y}\left(e_{i, j+\frac{1}{2}}^{n}\right)^{2}+\delta_{y}\left(e_{i, j-\frac{1}{2}}^{n}\right)^{2}\right] .
\end{aligned}
$$

Combining equation (3.4) and estimate (3.12), straightforward calculations result in

$$
\begin{aligned}
\left(e_{i j}^{*}\right)^{2} \leqslant & \left(e_{i j}^{n}\right)^{2}+k\left\{-\frac{\mu}{2 h}\left(e_{i, j+1}^{n}-e_{i, j-1}^{n}\right) e_{i j}^{n}+\frac{a}{h^{2}}\left(e_{i, j+1}^{n}-2 e_{i j}^{n}+e_{i, j-1}^{n}\right) e_{i j}^{n}+C\left(e_{i j}^{n}\right)^{2}\right\} \\
& +\frac{\mu^{2} k^{2}}{4 h^{2}}\left[\left(e_{i, j+1}^{n}\right)^{2}+\left(e_{i, j-1}^{n}\right)^{2}\right]+\frac{a^{2} k^{2}}{2 h^{2}}\left[\left(\delta_{y} e_{i, j+\frac{1}{2}}^{n}\right)^{2}+\left(\delta_{y} e_{i, j-\frac{1}{2}}^{n}\right)^{2}\right] \\
& +\frac{a \mu k^{2}}{4 h}\left[\left(\delta_{y} e_{i, j+\frac{1}{2}}^{n}\right)^{2}+\left(\delta_{y} e_{i, j-\frac{1}{2}}^{n}\right)^{2}\right]+\frac{C^{2} k^{2}}{4}\left(2+\frac{a}{h^{2}}\right)\left(e_{i j}^{n}\right)^{2}+\frac{3 a k^{2}}{4 h^{2}}\left[\left(e_{i, j+1}^{n}\right)^{2}+4\left(e_{i j}^{n}\right)^{2}+\left(e_{i, j-1}^{n}\right)^{2}\right] .
\end{aligned}
$$


Summing this up from $i, j=1,2, \ldots, M-1$, and rearranging of terms, we obtain

$$
\begin{aligned}
\sum_{i, j=1}^{M-1}\left(e_{i j}^{*}\right)^{2} \leqslant & \sum_{i, j=1}^{M-1}\left(e_{i j}^{n}\right)^{2}-\frac{\mu k}{2 h} \sum_{i, j=1}^{M-1}\left(e_{i, j+1}^{n}-e_{i, j-1}^{n}\right) e_{i j}^{n}+\frac{a k}{h^{2}} \sum_{i, j=1}^{M-1}\left(e_{i, j+1}^{n}-2 e_{i j}^{n}+e_{i, j-1}^{n}\right) e_{i j}^{n}+C k \sum_{i, j=1}^{M-1}\left(e_{i j}^{n}\right)^{2} \\
& +\frac{C^{2} k^{2}}{4}\left(2+\frac{a}{h^{2}}\right) \sum_{i, j=1}^{M-1}\left(e_{i j}^{n}\right)^{2}+\frac{\mu^{2} k^{2}}{4 h^{2}} \sum_{i, j=1}^{M-1}\left[\left(e_{i, j+1}^{n}\right)^{2}+\left(e_{i, j-1}^{n}\right)^{2}\right] \\
& +\frac{3 a k^{2}}{4 h^{2}} \sum_{i, j=1}^{M-1}\left[\left(e_{i, j+1}^{n}\right)^{2}+4\left(e_{i j}^{n}\right)^{2}+\left(e_{i, j-1}^{n}\right)^{2}\right]+\frac{a k^{2}}{2}\left(\frac{a}{h^{2}}+\frac{\mu}{2 h}\right) \sum_{i, j=1}^{M-1}\left[\left(\delta_{y} e_{i, j+\frac{1}{2}}^{n}\right)^{2}+\left(\delta_{y} e_{i, j-\frac{1}{2}}^{n}\right)^{2}\right],
\end{aligned}
$$

which implies

$$
\begin{aligned}
\sum_{i, j=1}^{M-1}\left(e_{i j}^{*}\right)^{2} \leqslant & \sum_{i, j=1}^{M-1}\left(e_{i j}^{n}\right)^{2}-\frac{\mu k}{2 h} \sum_{i, j=1}^{M-1}\left(e_{i, j+1}^{n}-e_{i, j-1}^{n}\right) e_{i j}^{n}+\frac{a k}{h^{2}} \sum_{i, j=1}^{M-1}\left(e_{i, j+1}^{n}-2 e_{i j}^{n}+e_{i, j-1}^{n}\right) e_{i j}^{n} \\
& +C k\left[1+\frac{\mu^{2} k}{2 C h^{2}}+\frac{C k}{4}\left(2+\frac{a}{h^{2}}\right)+\frac{9 a k}{2 C h^{2}}\right] \sum_{i, j=1}^{M-1}\left(e_{i j}^{n}\right)^{2} \\
& +\frac{a k}{2}\left(\frac{2 a k}{h^{2}}+\frac{\mu k}{h}\right) \sum_{i=1}^{M-1 M-1} \sum_{j=0}^{1}\left(\delta_{y} e_{i, j+\frac{1}{2}}^{n}\right)^{2} .
\end{aligned}
$$

Multiplying both sides of estimate (3.13) by $\mathrm{h}^{2}$, and using equations (2.36)-(2.37) of Lemma 2.1, it is not hard to see that

$$
\begin{aligned}
\left\|e^{*}\right\|_{L^{2}(\Omega)}^{2} \leqslant & \left\|e^{\mathfrak{n}}\right\|_{L^{2}(\Omega)}^{2}-a k\left\|\delta_{y} e^{\mathfrak{n}}\right\|_{L^{2}(\Omega)}^{2}+C k\left[1+\frac{\mu^{2} k}{2 C h^{2}}+\frac{C k}{4}\left(2+\frac{a}{h^{2}}\right)+\frac{9 a k}{2 C h^{2}}\right]\left\|e^{\mathfrak{n}}\right\|_{L^{2}(\Omega)}^{2} \\
& +\frac{a k}{2}\left(\frac{2 a k}{h^{2}}+\frac{\mu k}{h}\right)\left\|\delta_{y} e^{\mathfrak{n}}\right\|_{L^{2}(\Omega)}^{2} \\
= & \left\|e^{\mathfrak{n}}\right\|_{L^{2}(\Omega)}^{2}-a k\left[1-\frac{1}{2}\left(\frac{2 a k}{h^{2}}+\frac{\mu k}{h}\right)\right]\left\|\delta_{y} e^{\mathfrak{n}}\right\|_{L^{2}(\Omega)}^{2} \\
& +C k\left[1+\frac{\mu^{2} k}{2 C h^{2}}+\frac{C k}{4}\left(2+\frac{a}{h^{2}}\right)+\frac{9 a k}{2 C h^{2}}\right]\left\|e^{\mathfrak{n}}\right\|_{L^{2}(\Omega)}^{2} .
\end{aligned}
$$

From the time step requirement (2.33), i.e., $\max \left\{\frac{2 a k}{h^{2}}, \frac{|\mu| k}{h}\right\} \leqslant 1$, it is easy to see that $\frac{2 a k}{h^{2}}+\frac{\mu k}{h} \leqslant 2$, which is equivalent to $1-\frac{1}{2}\left(\frac{2 a k}{h^{2}}+\frac{\mu k}{h}\right) \geqslant 0$. This fact, together with estimate (3.14) provide

$$
\begin{aligned}
\left\|e^{*}\right\|_{L^{2}(\Omega)}^{2} & \leqslant\left\|e^{n}\right\|_{L^{2}(\Omega)}^{2}+C k\left[1+\frac{\mu^{2}}{4 a C}+\frac{C}{8}+\frac{9}{4 C}+\frac{C k}{2}\right]\left\|e^{n}\right\|_{L^{2}(\Omega)}^{2} \\
& =\left\|e^{\mathfrak{n}}\right\|_{L^{2}(\Omega)}^{2}+C k\left[g(C, \mu, a)+\frac{C k}{2}\right]\left\|e^{\mathfrak{n}}\right\|_{L^{2}(\Omega)}^{2}
\end{aligned}
$$

where

$$
g(C, \mu, a)=1+\frac{\mu^{2}}{4 a C}+\frac{C}{8}+\frac{9}{4 C} .
$$

Similarly, using equations (2.24), (2.27), and (2.30), adapting the proof for $\left\|e^{*}\right\|_{\mathrm{L}^{2}(\Omega)}^{2}$, by replacing $\frac{k}{2}$ by $k$, it not difficult to show that

$$
\left\|e^{* *}\right\|_{L^{2}(\Omega)}^{2} \leqslant\left\|e^{*}\right\|_{L^{2}(\Omega)}^{2}+2 \mathrm{Ck}[g(C, \mu, a)+C k]\left\|e^{*}\right\|_{L^{2}(\Omega)}^{2} .
$$


Finally, plugging equations (2.25), (2.27), and (2.31), following the proof for $\left\|e^{*}\right\|_{\mathrm{L}^{2}(\Omega)}^{2}$, but replacing $n$ and $*$ by $* *$ and $n+1$, respectively, it is easy to see that

$$
\left\|e^{\mathfrak{n}+1}\right\|_{\mathrm{L}^{2}(\Omega)}^{2} \leqslant\left\|e^{* *}\right\|_{\mathrm{L}^{2}(\Omega)}^{2}+\mathrm{Ck}\left[g(C, \mu, a)+\frac{\mathrm{Ck}}{2}\right]\left\|e^{* *}\right\|_{\mathrm{L}^{2}(\Omega)}^{2} .
$$

In what follows, we must find a relationship between $\left\|e^{n+1}\right\|_{L^{2}(\Omega)}^{2}$ and $\left\|e^{n}\right\|_{L^{2}(\Omega)}^{2}$. Combining estimates (3.15), (3.16), and (3.17), direct computations yield

$$
\begin{aligned}
\left\|e^{n+1}\right\|_{L^{2}(\Omega)}^{2} \leqslant & {\left[1+C k\left(g(C, \mu, a)+\frac{C k}{2}\right)\right]^{2}[1+2 C k(g(C, \mu, a)+C k)]\left\|e^{n}\right\|_{L^{2}(\Omega)}^{2} } \\
= & \left\{1+3 C g(C, \mu, a) k+C^{2}[3+5 g(C, \mu, a)] k^{2}+C^{3} g(C, \mu, a)\left[7+2 g(C, \mu, a)^{2}\right] k^{3}\right. \\
& \left.+C^{4}\left[\frac{9}{4}+4 g(C, \mu, a)^{2}\right] k^{4}+\frac{5}{2} C^{5} g(C, \mu, a) k^{5}+\frac{1}{2} C^{6} g(C, \mu, a) k^{6}\right\}\left\|e^{\mathfrak{n}}\right\|_{L^{2}(\Omega)}^{2} .
\end{aligned}
$$

Summing this up from $n=0,1,2, . ., p-1$, for any positive integer $p$ satisfying $1 \leqslant p \leqslant N$, it is easy to see that

$$
\begin{aligned}
\left\|e^{p}\right\|_{L^{2}(\Omega)}^{2} \leqslant & \left\|e^{0}\right\|_{L^{2}(\Omega)}^{2}+C k\left\{3 g(C, \mu, a)+C[3+5 g(C, \mu, a)] k+C^{2} g(C, \mu, a)\left[7+2 g(C, \mu, a)^{2}\right] k^{2}\right. \\
& \left.+C^{3}\left[\frac{9}{4}+4 g(C, \mu, a)^{2}\right] k^{3}+\frac{5}{2} C^{4} g(C, \mu, a) k^{4}+\frac{1}{2} C^{5} g(C, \mu, a) k^{5}\right\} \sum_{n=0}^{p-1}\left\|e^{n}\right\|_{L^{2}(\Omega)}^{2} .
\end{aligned}
$$

It comes from the initial condition given in (2.32) that $e_{i j}^{0}=0$, for $i, j=0,1, . ., M$. So $\left\|e^{0}\right\|_{L^{2}(\Omega)}^{2}=0$. Applying the Gronwall Lemma, estimate (3.18) provides

$$
\begin{aligned}
\left\|e^{p}\right\|_{L^{2}(\Omega)}^{2} \leqslant & \exp \left\{C k p \left[3 g(C, \mu, a)+C[3+5 g(C, \mu, a)] k+C^{2} g(C, \mu, a)\left[7+2 g(C, \mu, a)^{2}\right] k^{2}\right.\right. \\
& \left.\left.+C^{3}\left(\frac{9}{4}+4 g(C, \mu, a)^{2}\right) k^{3}+\frac{5}{2} C^{4} g(C, \mu, a) k^{4}+\frac{1}{2} C^{5} g(C, \mu, a) k^{5}\right]\right\} .
\end{aligned}
$$

But $p \leqslant N$ and $k=\frac{T}{N}$, so $C k p \leqslant C T$. A combination of this inequality together with estimate (3.19) gives

$$
\begin{aligned}
\left\|e^{\mathfrak{p}}\right\|_{L^{2}(\Omega)}^{2} \leqslant & \exp \left\{C T \left[3 g(C, \mu, a)+C[3+5 g(C, \mu, a)] k+C^{2} g(C, \mu, a)\left[7+2 g(C, \mu, a)^{2}\right] k^{2}\right.\right. \\
& \left.\left.+C^{3}\left(\frac{9}{4}+4 g(C, \mu, a)^{2}\right) k^{3}+\frac{4}{2} C^{4} g(C, \mu, a) k^{4}+\frac{1}{2} C^{5} g(C, \mu, a) k^{5}\right]\right\} \\
\leqslant & \exp \left\{7 C \operatorname{CTg}(C, \mu, a)(1+g(C, \mu, a))^{2} \sum_{l=0}^{5}(C k)^{l}\right\} .
\end{aligned}
$$

Taking the square root, it holds

$$
\left\|e^{\mathfrak{p}}\right\|_{L^{2}(\Omega)} \leqslant \exp \left\{\frac{7 C T}{2} g(C, \mu, a)(1+g(C, \mu, a))^{2} \sum_{l=0}^{5}(C k)^{l}\right\} .
$$

Since $\left\|\phi^{\mathfrak{p}}\right\|_{\mathrm{L}^{2}(\Omega)}-\left\|\bar{\phi}^{\mathrm{p}}\right\|_{\mathrm{L}^{2}(\Omega)} \leqslant\left\|\phi^{\mathfrak{p}}-\bar{\phi}^{\mathrm{p}}\right\|_{\mathrm{L}^{2}(\Omega)}=\left\|e^{\mathfrak{p}}\right\|_{\mathrm{L}^{2}(\Omega)}$, estimate (3.20) implies

$$
\left\|\phi^{p}\right\|_{L^{2}(\Omega)} \leqslant\left\|\bar{\phi}^{p}\right\|_{L^{2}(\Omega)}+\exp \left\{\frac{7 C T}{2} g(C, \mu, a)(1+g(C, \mu, a))^{2} \sum_{l=0}^{5}(C k)^{l}\right\} .
$$

This completes the proof of Theorem 3.1 thanks to estimate (2.34). 


\section{Error estimate of the numerical scheme}

This section deals with the error estimates of the three-level explicit time-split MacCormack method (2.29)-(2.32) applied to the nonlinear convection-diffusion-reaction problem (1.1)-(1.3). We assume that the analytical solution $\bar{\phi}$ satisfies inequality (2.34).

Firstly, we introduce the following discrete norms

$$
\||\phi|\|_{\mathrm{L}^{\infty}\left(0, \mathrm{~T} ; \mathrm{L}^{2}(\Omega)\right)}=\max _{0 \leqslant n \leqslant N}\left\|\phi^{\mathrm{n}}\right\|_{\mathrm{L}^{2}(\Omega)}, \quad\||\phi|\|_{\mathrm{L}^{2}\left(0, \mathrm{~T} ; \mathrm{L}^{2}(\Omega)\right)}=\left(k \sum_{n=0}^{\mathrm{N}}\left\|\phi^{\mathrm{n}}\right\|_{\mathrm{L}^{2}(\Omega)}^{2}\right)^{\frac{1}{2}},
$$

and

$$
\||\phi|\|_{L^{1}\left(0, T ; L^{2}(\Omega)\right)}=k \sum_{n=0}^{N}\left\|\phi^{n}\right\|_{L^{2}(\Omega)} \text {, for } \phi \in \mathcal{U}_{h},
$$

where $\mathcal{U}_{h}=\left\{\phi_{i j}^{n}, 0 \leqslant n \leqslant N ; 0 \leqslant i, j \leqslant M\right\}$, is the space of grid functions defined on $\Omega_{h} \times \Omega_{k}$. This discrete space is defined in Section 2.

Theorem 4.1. Suppose $\phi$ be the approximate solution provided by the three-level time-split MacCormack method (2.29)-(2.32). Under the time step requirement (2.33), the error term $e=\phi-\bar{\phi}$, verifies

$$
\|\mid\|_{\mathrm{L}^{\infty}\left(0, \mathrm{~T} ; \mathrm{L}^{2}(\Omega)\right)} \leqslant\left(1+k+k^{2}\right)^{2} \sqrt{\mathrm{CT} \psi(k, h)} \exp \left\{\frac{\mathrm{CT}}{2}\left(1+k+k^{2}\right)^{5}\right\}\left(k+h^{4}\right),
$$

where $\mathrm{C}>0$, is a constant that does not depend on the time step $\mathrm{k}$ and the grid spacing $\mathrm{h}$, and $\psi(\mathrm{k}, \mathrm{h})=$ $1+k^{2}+k^{3}+h^{2}+(1+k)\left(1+h^{4}+h^{8}\right)$.

The proof of Theorem 4.1 requires some intermediate results (Lemmas 2.1, 4.2, and 4.3).

Lemma 4.2. Suppose $v \in \mathrm{H}^{4}(\Omega)$, be a function that satisfies $\left.v\right|_{\left[x_{i}, x_{i+1}\right]} \in \mathcal{C}^{6}\left[x_{i}, x_{i+1}\right]$. Then, it holds

$$
\begin{aligned}
\frac{1}{h}\left(v_{i+1}-v_{i}\right) & =v_{x, i}+\frac{h}{2} v_{2 x, i}+\frac{h^{2}}{6} v_{3 x, i}+\cdots+\frac{h^{5}}{720} v_{6 x}\left(\theta_{i}^{(3)}\right), \text { for } i=0,1, \ldots, M-1, \\
\frac{1}{h}\left(v_{2 x, i+1}-v_{2 x, i}\right) & =v_{3 x, i}+\frac{h}{2} v_{4 x, i}+\frac{h^{2}}{6} v_{5 x, i}+\frac{h^{3}}{720} v_{6 x}\left(\theta_{i}^{(3)}\right), \text { for } i=0,1, \ldots, M-1, \\
\frac{1}{h^{2}}\left(v_{i+1}-2 v_{i}+v_{i-1}\right)-v_{2 x, i} & =\frac{h^{2}}{12} v_{4 x, i}-\frac{h^{4}}{720}\left[v_{6 x}\left(\theta_{i}^{(3)}\right)+v_{6 x}\left(\theta_{i}^{(4)}\right)\right], \text { for } i=1,2, \ldots, M-1,
\end{aligned}
$$

where $\theta_{i}^{(4)} \in\left(x_{i-1}, x_{i}\right), \theta_{i}^{(3)} \in\left(x_{i}, x_{i+1}\right)$ and $v_{m x}$ denotes the derivative of order $m$ of $v$. Furthermore, for $i=2,3, \ldots, M-2$,

$$
\begin{aligned}
& \frac{1}{\mathrm{~h}^{4}}\left(v_{i+2}-4 v_{i+1}+6 v_{i}-4 v_{i-1}+v_{i-2}\right)-v_{4 x, i}=h^{2}\left\{\frac{1}{720}\left[v_{6 x}\left(\theta_{i}^{(1)}\right)+v_{6 x}\left(\theta_{i}^{(2)}\right)\right]+\frac{241}{3220}\left[v_{6 x}\left(\theta_{i}^{(3)}\right)+v_{6 x}\left(\theta_{i}^{(4)}\right)\right]\right\}, \\
& \text { where } \theta_{i}^{(2)} \in\left(x_{i-2}, x_{i-1}\right), \theta_{i}^{(4)} \in\left(x_{i-1}, x_{i}\right), \theta_{i}^{(3)} \in\left(x_{i}, x_{i+1}\right) \text { and } \theta_{i}^{(1)} \in\left(x_{i+1}, x_{i+2}\right) .
\end{aligned}
$$

Proof. The proof of the first equation is obvious according to the Taylor series expansion about $x_{i}$ using forward difference representation. For the proof of the other equations, we refer the readers to [27].

Lemma 4.3. The terms $\rho_{i j}^{\alpha}$ and $\widehat{\rho}_{i j}^{n}$ given by equations (2.8) and (2.15), respectively, can be bounded as

$$
\left|\rho_{i j}^{n}\right|,\left|\rho_{i j}^{\bar{*}}\right|,\left|\widehat{\rho}_{i j}^{n}\right| \leqslant \widehat{C}_{1}\left[1+\widehat{C}_{2} h^{2}+\widehat{C}_{3} h^{4}\right],
$$

where $\widehat{C}_{l}, l=1,2,3$, are positive constant independent of the time step $k$ and the grid spacing $h$. 
Proof. It comes from equation (2.8) that

$$
\begin{aligned}
\rho_{i j}^{n}= & \frac{1}{8}\left\{a^{2} \delta_{y}^{2}\left(\delta_{y}^{2} \bar{\phi}_{i j}^{n}\right)-2 a \mu \delta_{y}^{2}\left(\delta_{y} \bar{\phi}_{i, j+\frac{1}{2}}^{n}\right)+\mu^{2} \delta_{y}^{2} \bar{\phi}_{i j}^{n}-\mu \delta_{y}\left(f\left(\bar{\phi}_{i, j+\frac{1}{2}}^{n}\right)\right)+a \delta_{y}^{2}\left(f\left(\bar{\phi}_{i j}^{n}\right)\right)\right. \\
& \left.+\left[-\mu \delta_{y} \bar{\phi}_{i, j+\frac{1}{2}}^{n}+a \delta_{y}^{2} \bar{\phi}_{i j}^{n}+f\left(\bar{\phi}_{i j}^{n}\right)\right] f^{\prime}\left(\bar{\phi}_{i j}^{n}\right)\right\}
\end{aligned}
$$

which is equivalent to

$$
\begin{aligned}
\rho_{i j}^{n}= & \frac{1}{8}\left\{\frac{\mu^{2}}{h^{2}}\left(\bar{\phi}_{i, j+1}^{n}-2 \bar{\phi}_{i j}^{n}+\bar{\phi}_{i, j-1}^{n}\right)-\frac{2 a \mu}{h^{3}}\left[\left(\bar{\phi}_{i, j+2}^{n}-2 \bar{\phi}_{i, j+1}^{n}+\bar{\phi}_{i j}^{n}\right)-\left(\bar{\phi}_{i, j+1}^{n}-2 \bar{\phi}_{i j}^{n}+\bar{\phi}_{i, j-1}^{n}\right)\right]\right. \\
& +\frac{a^{2}}{h^{4}}\left(\bar{\phi}_{i, j+2}^{n}-4 \bar{\phi}_{i, j+1}^{n}+6 \bar{\phi}_{i j}^{n}-4 \bar{\phi}_{i, j-1}^{n}+\bar{\phi}_{i, j-2}^{n}\right)-\frac{\mu}{h}\left[f\left(\bar{\phi}_{i, j+1}^{n}\right)-f\left(\bar{\phi}_{i j}^{n}\right)\right]+\frac{a}{h^{2}}\left[f\left(\bar{\phi}_{i, j+1}^{n}\right)-2 f\left(\bar{\phi}_{i j}^{n}\right)\right. \\
& \left.\left.+f\left(\bar{\phi}_{i, j-1}^{n}\right)\right]+\left[-\frac{\mu}{h}\left(\bar{\phi}_{i, j+1}^{n}-\bar{\phi}_{i j}^{n}\right)+\frac{a}{h^{2}}\left(\bar{\phi}_{i, j+1}^{n}-2 \bar{\phi}_{i j}^{n}+\bar{\phi}_{i, j-1}^{n}\right)+f\left(\bar{\phi}_{i j}^{n}\right)\right] f^{\prime}\left(\bar{\phi}_{i j}^{n}\right)\right\} .
\end{aligned}
$$

This fact together with Lemma 4.2, result in

$$
\begin{aligned}
\rho_{i j}^{n}= & \frac{1}{8}\left\{\mu^{2} \bar{\phi}_{2 y, i j}^{n}+\frac{\mu^{2} h^{2}}{12} \bar{\phi}_{4 y, i j}^{n}-\frac{\mu^{2} h^{4}}{720}\left[\bar{\phi}_{6 y}^{n}\left(x_{i}, \theta_{j}^{(3)}\right)+\bar{\phi}_{6 y}^{n}\left(x_{i}, \theta_{j}^{(4)}\right)\right]-\frac{2 a \mu}{h}\left[\bar{\phi}_{2 y, i, j+1}^{n}-\bar{\phi}_{2 y, i j}^{n}\right.\right. \\
& \left.+\frac{h^{2}}{12}\left(\bar{\phi}_{4 y, i, j+1}^{n}-\bar{\phi}_{4 y, i j}^{n}\right)-\frac{h^{4}}{720}\left(\left(\bar{\phi}_{6 y}^{n}\left(x_{i}, \theta_{j+1}^{(3)}\right)+\bar{\phi}_{6 y}^{n}\left(x_{i}, \theta_{j+1}^{(4)}\right)\right)-\left(\bar{\phi}_{6 y}^{n}\left(x_{i}, \theta_{j}^{(3)}\right)+\bar{\phi}_{6 y}^{n}\left(x_{i}, \theta_{j}^{(4)}\right)\right)\right)\right] \\
& +a^{2}\left[\bar{\phi}_{4 y, i j}^{n}+h^{2}\left(\frac{1}{720}\left[\bar{\phi}_{6 y}^{n}\left(x_{i}, \theta_{j}^{(1)}\right)+\bar{\phi}_{6 y}^{n}\left(x_{i}, \theta_{j}^{(2)}\right)\right]+\frac{241}{3220}\left[\bar{\phi}_{6 y}^{n}\left(x_{i}, \theta_{j}^{(3)}\right)+\bar{\phi}_{6 y}^{n}\left(x_{i}, \theta_{j}^{(4)}\right)\right]\right)\right] \\
& \left.\left.\left.\left.\left.\left.-\mu[f o \bar{\phi})_{y, i j}^{n}+\frac{h}{2} f o \bar{\phi}\right)_{2 y, i j}^{n}+\frac{h^{2}}{6} f o \bar{\phi}\right)_{3 y, i j}^{n}+\frac{h^{3}}{24} f o \bar{\phi}\right)_{4 y, i j}^{n}+\frac{h^{4}}{120} f o \bar{\phi}\right)_{5 y, i j}^{n}+\frac{h^{5}}{720} f o \bar{\phi}\right)_{6 y}^{n}\left(x_{i}, \theta_{j}^{(3)}\right)\right] \\
& \left.\left.\left.\left.\times a[f o \bar{\phi})_{2 y, i j}^{n}+\frac{h^{2}}{12} f o \bar{\phi}\right)_{4 y, i j}^{n}-\frac{h^{4}}{720}(f o \bar{\phi})_{6 y}^{n}\left(x_{i}, \theta_{j}^{(3)}\right)+f o \bar{\phi}\right)_{6 y}^{n}\left(x_{i}, \theta_{j}^{(4)}\right)\right)\right]+\left[-\mu\left(\bar{\phi}_{y, i j}^{n}+\frac{h}{2} \bar{\phi}_{2 y, i j}^{n}\right.\right. \\
& \left.+\frac{h^{2}}{6} \bar{\phi}_{3 y, i j}^{n}+\frac{h^{3}}{24} \bar{\phi}_{4 y, i j}^{n}+\frac{h^{4}}{120} \bar{\phi}_{5 y, i j}^{n}+\frac{h^{5}}{720} \bar{\phi}_{6 y}^{n}\left(x_{i}, \theta_{j}^{(3)}\right)\right) \\
& \left.\left.+a\left(\bar{\phi}_{2 y, i j}^{n}+\frac{h^{2}}{12} \bar{\phi}_{4 y, i j}^{n}-\frac{h^{4}}{720}\left(\bar{\phi}_{6 y}^{n}\left(x_{i}, \theta_{j}^{(3)}\right)+\bar{\phi}_{6 y}^{n}\left(x_{i}, \theta_{j}^{(4)}\right)\right)\right) f^{\prime}\left(\bar{\phi}_{i j}^{n}\right)\right]+f\left(\bar{\phi}_{i j}^{n}\right) f^{\prime}\left(\bar{\phi}_{i j}^{n}\right)\right\} .
\end{aligned}
$$

Since $\left.\bar{\phi}(x, \cdot, t)\right|_{\left[y_{j}, y_{j+1}\right]}$, fo $\left.\bar{\phi}(x, \cdot, t)\right|_{\left[y_{j}, y_{j+1}\right]} \in \mathcal{C}^{6}\left(\left[y_{j}, y_{j+1}\right]\right)$, for every $x \in(0,1), t \in(0, T)$ and $j=0,1,2, \ldots$, $M-1,\||\bar{\phi}|\|_{\mathrm{L}^{\infty}\left(0, \mathrm{~T} ; \mathrm{L}^{2}(\Omega)\right)} \leqslant \widetilde{\mathrm{C}}$ (according to estimate (2.34)), $\mathrm{f}^{\prime}$ (the derivative of $\mathrm{f}$ ) is continuous and $h \leqslant 1+h^{2}$, taking the modulus of $\rho_{i j}^{n}$, there exist positive constants $\widehat{C}_{l}, l=1,2,3$, which do not depend on the time step $k$ and the mesh grid $h$ such that

$$
\left|\rho_{i j}^{n}\right| \leqslant \widehat{C}_{1}\left[1+\widehat{C}_{2} h^{2}+\widehat{C}_{3} h^{4}\right], \text { for } n=0,1, \ldots, N \text { and } i, j=0,1, \ldots, M .
$$

In way similar, one proves the other estimates. This completes the proof of Lemma 4.3.

Using Lemmas 2.1, 4.2, and 4.3, we are ready to prove Theorem 4.1.

Proof of Theorem 4.1. Firstly, it is worth noticing to recall that the error term provided by the time-split method (2.29)-(2.32) is given by $e_{i j}^{n}=\phi_{i j}^{n}-\bar{\phi}_{i j}^{n}$, where $\bar{\phi}$ is the exact solution of equations (2.16), (2.24), and (2.25) and $\phi$ represents the numerical solution given by the algorithm (2.29)-(2.32).

From equation (3.2), we know that

$$
e_{i j}^{*}=e_{i j}^{n}+\frac{k}{2}\left(\frac{a}{h^{2}}\left(e_{i, j+1}^{n}-2 e_{i j}^{n}+e_{i, j-1}^{n}\right)-\frac{\mu}{2 h}\left(e_{i, j+1}^{n}-e_{i, j-1}^{n}\right)+f\left(\phi_{i j}^{n}\right)-f\left(\bar{\phi}_{i j}^{n}\right)\right)+\frac{k^{2}}{2}\left(\rho_{i j}^{n}+\widehat{\rho}_{i j}^{n}+\rho_{i j}^{\bar{*}}\right)
$$




$$
+\mathrm{O}\left(\mathrm{k}^{3}+k h^{2}\right)
$$

which can be written as

$$
\begin{aligned}
e_{i j}^{*}= & e_{i j}^{n}+\frac{k}{2}\left(\frac{a}{h^{2}}\left(e_{i, j+1}^{n}-2 e_{i j}^{n}+e_{i, j-1}^{n}\right)-\frac{\mu}{2 h}\left(e_{i, j+1}^{n}-e_{i, j-1}^{n}\right)+f\left(\phi_{i j}^{n}\right)-f\left(\bar{\phi}_{i j}^{n}\right)\right)+\frac{k^{2}}{2}\left(\rho_{i j}^{n}+\widehat{\rho}_{i j}^{n}+\rho_{i j}^{\bar{*}}\right) \\
& +C_{r}\left(k^{3}+k h^{2}\right),
\end{aligned}
$$

where $C_{r}$ is a parameter which does not depend on the time step $k$ and the grid spacing $h$, and whose value can change from place to place, $\rho_{i j}^{\alpha}$ and $\widehat{\rho}_{i j}^{n}$ are given by equations (2.8) and (2.15), respectively. Performing simple calculations, it is not hard to see that

$$
\begin{aligned}
\left(e_{i j}^{*}\right)^{2}= & \left(e_{i j}^{n}\right)^{2}+k\left\{\frac{a}{h^{2}}\left(e_{i, j+1}^{n}-2 e_{i j}^{n}+e_{i, j-1}^{n}\right) e_{i j}^{n}-\frac{\mu}{2 h}\left(e_{i, j+1}^{n}-e_{i, j-1}^{n}\right) e_{i j}^{n}+\left(f\left(\phi_{i j}^{n}\right)-f\left(\bar{\phi}_{i j}^{n}\right)\right) e_{i j}^{n}\right\} \\
& +k^{2}\left(\rho_{i j}^{n}+\widehat{\rho}_{i j}^{n}+\rho_{i j}^{\bar{*}}\right) e_{i j}^{n}+2 C_{r}\left(k^{3}+k h^{2}\right) e_{i j}^{n}+\frac{k^{3}}{2}\left[\frac{a}{h^{2}}\left(e_{i, j+1}^{n}-2 e_{i j}^{n}+e_{i, j-1}^{n}\right)\right. \\
& \left.-\frac{\mu}{2 h}\left(e_{i, j+1}^{n}-e_{i, j-1}^{n}\right)+f\left(\phi_{i j}^{n}\right)-f\left(\bar{\phi}_{i j}^{n}\right)\right]\left(\rho_{i j}^{n}+\widehat{\rho}_{i j}^{n}+\rho_{i j}^{\bar{F}}\right)+C_{r} k^{2}\left(\rho_{i j}^{n}+\widehat{\rho}_{i j}^{n}+\rho_{i j}^{\bar{*}}\right)\left(k^{3}+k h^{2}\right) \\
& +\frac{k^{4}}{4}\left(\rho_{i j}^{n}+\widehat{\rho}_{i j}^{n}+\rho_{i j}^{\bar{k}}\right)^{2}+C_{r} k\left[\frac{a}{h^{2}}\left(e_{i, j+1}^{n}-2 e_{i j}^{n}+e_{i, j-1}^{n}\right)\right. \\
& \left.-\frac{\mu}{2 h}\left(e_{i, j+1}^{n}-e_{i, j-1}^{n}\right)+f\left(\phi_{i j}^{n}\right)-f\left(\bar{\phi}_{i j}^{n}\right)\right]\left(k^{3}+k h^{2}\right)+C_{r}^{2}\left(k^{3}+k h^{2}\right)^{2} \\
& +\frac{k^{2}}{4}\left[\frac{a}{h^{2}}\left(e_{i, j+1}^{n}-2 e_{i j}^{n}+e_{i, j-1}^{n}\right)-\frac{\mu}{2 h}\left(e_{i, j+1}^{n}-e_{i, j-1}^{n}\right)+f\left(\phi_{i j}^{n}\right)-f\left(\bar{\phi}_{i j}^{n}\right)\right]^{2} .
\end{aligned}
$$

Applying the inequalities: $(a \pm b \pm c)^{2} \leqslant 3\left(a^{2}+b^{2}+c^{2}\right),(a \pm b)^{2} \leqslant 2\left(a^{2}+b^{2}\right)$ and $2 a b \leqslant a^{2}+b^{2}$, for every $a, b, c \in \mathbb{R}$, together with the time step restriction (2.33) (that is, $2 a k \leqslant h^{2}$ and $|\mu| k \leqslant h$ ), equation (4.2) provides

$$
\begin{aligned}
\left(e_{i j}^{*}\right)^{2} \leqslant & \left(e_{i j}^{n}\right)^{2}+k\left\{\frac{a}{h^{2}}\left(e_{i, j+1}^{n}-2 e_{i j}^{n}+e_{i, j-1}^{n}\right) e_{i j}^{n}-\frac{\mu}{2 h}\left(e_{i, j+1}^{n}-e_{i, j-1}^{n}\right) e_{i j}^{n}+\left(f\left(\phi_{i j}^{n}\right)-f\left(\bar{\phi}_{i j}^{n}\right)\right) e_{i j}^{n}\right\} \\
& +\frac{5 k^{3}}{4}\left(\rho_{i j}^{n}+\widehat{\rho}_{i j}^{n}+\rho_{i j}^{\bar{*}}\right)^{2}+\frac{5 k}{4}\left(e_{i j}^{n}\right)^{2}+2 C_{r}^{2}\left(k^{\frac{5}{2}}+k^{\frac{1}{2}} h^{2}\right)^{2}+\frac{k^{4}}{2}\left(\rho_{i j}^{n}+\hat{\rho}_{i j}^{n}+\rho_{i j}^{\bar{k}}\right)^{2}+2 C_{r}^{2}\left(k^{3}+k h^{2}\right)^{2} \\
& +\frac{3 k^{3}}{2}\left[\frac{a^{2}}{h^{4}}\left(e_{i, j+1}^{n}-2 e_{i j}^{n}+e_{i, j-1}^{n}\right)^{2}+\frac{\mu^{2}}{4 h^{2}}\left(e_{i, j+1}^{n}-e_{i, j-1}^{n}\right)^{2}+\left(f\left(\phi_{i j}^{n}\right)-f\left(\bar{\phi}_{i j}^{n}\right)\right)^{2}\right]+\frac{k^{2}}{4}\left[\frac { a ^ { 2 } } { h ^ { 4 } } \left(e_{i, j+1}^{n}\right.\right. \\
& \left.-2 e_{i j}^{n}+e_{i, j-1}^{n}\right)^{2}+\frac{\mu^{2}}{4 h^{2}}\left(e_{i, j+1}^{n}-e_{i, j-1}^{n}\right)^{2}+\left(f\left(\phi_{i j}^{n}\right)-f\left(\bar{\phi}_{i j}^{n}\right)\right)^{2}-\frac{a \mu}{h^{3}}\left(e_{i, j+1}^{n}-e_{i, j-1}^{n}\right)\left(e_{i, j+1}^{n}\right. \\
& \left.\left.-2 e_{i j}^{n}+e_{i, j-1}^{n}\right)-\frac{\mu}{h}\left(e_{i, j+1}^{n}-e_{i, j-1}^{n}\right)\left(f\left(\phi_{i j}^{n}\right)-f\left(\bar{\phi}_{i j}^{n}\right)\right)+\frac{2 a}{h^{2}}\left(e_{i, j+1}^{n}-2 e_{i j}^{n}+e_{i, j-1}^{n}\right)\left(f\left(\phi_{i j}^{n}\right)-f\left(\bar{\phi}_{i j}^{n}\right)\right)\right],
\end{aligned}
$$

which implies

$$
\begin{aligned}
\left(e_{i j}^{*}\right)^{2} \leqslant & \left(e_{i j}^{n}\right)^{2}+k\left\{\frac{a}{h^{2}}\left(e_{i, j+1}^{n}-2 e_{i j}^{n}+e_{i, j-1}^{n}\right) e_{i j}^{n}-\frac{\mu}{2 h}\left(e_{i, j+1}^{n}-e_{i, j-1}^{n}\right) e_{i j}^{n}+\left(f\left(\phi_{i j}^{n}\right)-f\left(\bar{\phi}_{i j}^{n}\right)\right) e_{i j}^{n}\right\} \\
& +\frac{15 k^{3}}{4}\left[\left(\rho_{i j}^{n}\right)^{2}+\left(\widehat{\rho}_{i j}^{n}\right)^{2}+\left(\rho_{i j}^{*}\right)^{2}\right]+\frac{5 k}{4}\left(e_{i j}^{n}\right)^{2}+4 C_{r}^{2}\left(k^{5}+k h^{4}\right)+\frac{3 k^{4}}{2}\left[\left(\rho_{i j}^{n}\right)^{2}+\left(\widehat{\rho}_{i j}^{n}\right)^{2}+\left(\rho_{i j}^{*}\right)^{2}\right] \\
& +\frac{3 k^{3}}{2}\left[\frac{3 a^{2}}{h^{4}}\left[\left(e_{i, j+1}^{n}\right)^{2}+4\left(e_{i j}^{n}\right)^{2}+\left(e_{i, j-1}^{n}\right)^{2}\right]+\frac{\mu^{2}}{2 h^{2}}\left[\left(e_{i, j+1}^{n}\right)^{2}+\left(e_{i, j-1}^{n}\right)^{2}\right]+\left(f\left(\phi_{i j}^{n}\right)-f\left(\bar{\phi}_{i j}^{n}\right)\right)^{2}\right] \\
& +\frac{k^{2}}{4}\left\{\frac{2 a^{2}}{h^{2}}\left[\left(\delta_{y} e_{i, j+\frac{1}{2}}^{n}\right)^{2}+\left(\delta_{y} e_{i, j-\frac{1}{2}}^{n}\right)^{2}\right]+\frac{\mu^{2}}{2 h^{2}}\left[\left(e_{i, j+1}^{n}\right)^{2}+\left(e_{i, j-1}^{n}\right)^{2}\right]+\left(f\left(\phi_{i j}^{n}\right)-f\left(\bar{\phi}_{i j}^{n}\right)\right)^{2}\right. \\
& -\frac{a \mu}{h^{3}}\left(e_{i, j+1}^{n}-e_{i, j-1}^{n}\right)\left(e_{i, j+1}^{n}-2 e_{i j}^{n}+e_{i, j-1}^{n}\right)-\frac{\mu}{h}\left(e_{i, j+1}^{n}-e_{i, j-1}^{n}\right)\left(f\left(\phi_{i j}^{n}\right)-f\left(\bar{\phi}_{i j}^{n}\right)\right) \\
& \left.+\frac{a}{h^{2}}\left(e_{i, j+1}^{n}-2 e_{i j}^{n}+e_{i, j-1}^{n}\right)^{2}+\frac{a}{h^{2}}\left(f\left(\phi_{i j}^{n}\right)-f\left(\bar{\phi}_{i j}^{n}\right)\right)^{2}\right\}+4 C_{r}^{2}\left(k^{6}+k^{2} h^{4}\right) .
\end{aligned}
$$


Utilizing estimates (3.5)-(3.11), this becomes

$$
\begin{aligned}
\left(e_{i j}^{*}\right)^{2} \leqslant & \left(e_{i j}^{n}\right)^{2}+k\left\{\frac{a}{h^{2}}\left(e_{i, j+1}^{n}-2 e_{i j}^{n}+e_{i, j-1}^{n}\right) e_{i j}^{n}-\frac{\mu}{2 h}\left(e_{i, j+1}^{n}-e_{i, j-1}^{n}\right) e_{i j}^{n}+C\left(e_{i j}^{n}\right)^{2}\right\} \\
& +\frac{3 k^{3}}{2}\left(\frac{5}{2}+k\right)\left[\left(\rho_{i j}^{n}\right)^{2}+\left(\hat{\rho}_{i j}^{n}\right)^{2}+\left(\rho_{i j}^{\bar{F}}\right)^{2}\right]+4 C_{r}^{2} k\left(k^{5}+k^{4}+k h^{4}+h^{4}\right)+\frac{5 k}{4}\left(e_{i j}^{n}\right)^{2} \\
& +\frac{3 k^{3}}{2}\left[\frac{3 a^{2}}{h^{4}}\left[\left(e_{i, j+1}^{n}\right)^{2}+4\left(e_{i j}^{n}\right)^{2}+\left(e_{i, j-1}^{n}\right)^{2}\right]+\frac{\mu^{2}}{2 h^{2}}\left[\left(e_{i, j+1}^{n}\right)^{2}+\left(e_{i, j-1}^{n}\right)^{2}\right]+C^{2}\left(e_{i j}^{n}\right)^{2}\right] \\
& +\frac{k^{2}}{4}\left\{\frac{2 a^{2}}{h^{2}}\left[\left(\delta_{y} e_{i, j+\frac{1}{2}}^{n}\right)^{2}+\left(\delta_{y} e_{i, j-\frac{1}{2}}^{n}\right)^{2}\right]+\frac{\mu^{2}}{2 h^{2}}\left[\left(e_{i, j+1}^{n}\right)^{2}+\left(e_{i, j-1}^{n}\right)^{2}\right]+C^{2}\left(e_{i j}^{n}\right)^{2}-\frac{a \mu}{h}\left[\left(\delta_{y} e_{i, j+\frac{1}{2}}^{n}\right)^{2}\right.\right. \\
& \left.\left.-\left(\delta_{y} e_{i, j-\frac{1}{2}}^{n}\right)^{2}\right]+\frac{\mu^{2}}{4 h^{2}}\left(e_{i, j+1}^{n}-e_{i, j-1}^{n}\right)^{2}+C^{2}\left(e_{i j}^{n}\right)^{2}+\frac{3 a}{h^{2}}\left[\left(e_{i, j+1}^{n}\right)^{2}+4\left(e_{i j}^{n}\right)^{2}+\left(e_{i, j-1}^{n}\right)^{2}\right]+\frac{a C^{2}}{h^{2}}\left(e_{i j}^{n}\right)^{2}\right\} .
\end{aligned}
$$

Since $k h^{4} \leqslant k^{2}+h^{8}$, this implies

$$
\begin{aligned}
\left(e_{i j}^{*}\right)^{2} \leqslant & \left(e_{i j}^{n}\right)^{2}+k\left\{\frac{a}{h^{2}}\left(e_{i, j+1}^{n}-2 e_{i j}^{n}+e_{i, j-1}^{n}\right) e_{i j}^{n}-\frac{\mu}{2 h}\left(e_{i, j+1}^{n}-e_{i, j-1}^{n}\right) e_{i j}^{n}+C\left(e_{i j}^{n}\right)^{2}\right\} \\
& +\frac{3 k^{3}}{2}\left(\frac{5}{2}+k\right)\left[\left(\rho_{i j}^{n}\right)^{2}+\left(\hat{\rho}_{i j}^{n}\right)^{2}+\left(\rho_{i j}^{\bar{x}}\right)^{2}\right]+4 C_{r}^{2} k\left(k^{2}+k^{4}+k^{5}+h^{4}+h^{8}\right)+\frac{5 k}{4}\left(e_{i j}^{n}\right)^{2} \\
& +\frac{3 k^{3}}{2}\left[\frac{3 a^{2}}{h^{4}}\left[\left(e_{i, j+1}^{n}\right)^{2}+4\left(e_{i j}^{n}\right)^{2}+\left(e_{i, j-1}^{n}\right)^{2}\right]+\frac{\mu^{2}}{2 h^{2}}\left[\left(e_{i, j+1}^{n}\right)^{2}+\left(e_{i, j-1}^{n}\right)^{2}\right]+C^{2}\left(e_{i j}^{n}\right)^{2}\right] \\
& +\frac{k^{2}}{4}\left\{\frac{2 a^{2}}{h^{2}}\left[\left(\delta_{y} e_{i, j+\frac{1}{2}}^{n}\right)^{2}+\left(\delta_{y} e_{i, j-\frac{1}{2}}^{n}\right)^{2}\right]+\frac{\mu^{2}}{2 h^{2}}\left[\left(e_{i, j+1}^{n}\right)^{2}+\left(e_{i, j-1}^{n}\right)^{2}\right]+C^{2}\left(e_{i j}^{n}\right)^{2}-\frac{a \mu}{h}\left[\left(\delta_{y} e_{i, j+\frac{1}{2}}^{n}\right)^{2}\right.\right. \\
& \left.\left.-\left(\delta_{y} e_{i, j-\frac{1}{2}}^{n}\right)^{2}\right]+\frac{\mu^{2}}{4 h^{2}}\left(e_{i, j+1}^{n}-e_{i, j-1}^{n}\right)^{2}+C^{2}\left(e_{i j}^{n}\right)^{2}+\frac{3 a}{h^{2}}\left[\left(e_{i, j+1}^{n}\right)^{2}+4\left(e_{i j}^{n}\right)^{2}+\left(e_{i, j-1}^{n}\right)^{2}\right]+\frac{a C^{2}}{h^{2}}\left(e_{i j}^{n}\right)^{2}\right\} .
\end{aligned}
$$

Now, using the time step restriction (2.33) together with Lemma 4.3, straightforward computations give

$$
\begin{aligned}
\left(e_{i j}^{*}\right)^{2} \leqslant & \left(e_{i j}^{n}\right)^{2}+k\left\{\frac{a}{h^{2}}\left(e_{i, j+1}^{n}-2 e_{i j}^{n}+e_{i, j-1}^{n}\right) e_{i j}^{n}-\frac{\mu}{2 h}\left(e_{i, j+1}^{n}-e_{i, j-1}^{n}\right) e_{i j}^{n}+C\left(e_{i j}^{n}\right)^{2}\right\} \\
& +\frac{9 \widehat{C}_{1}^{2} k^{3}}{2}\left(\frac{5}{2}+k\right)\left[1+\widehat{C}_{2} h^{2}+\widehat{C}_{3} h^{4}\right]^{2}+4 C_{r}^{2} k\left(k^{2}+k^{4}+k^{5}+h^{4}+h^{8}\right)+\frac{5 k}{4}\left(e_{i j}^{n}\right)^{2} \\
& +\frac{3 k}{2}\left[\frac{3}{4}\left[\left(e_{i, j+1}^{n}\right)^{2}+4\left(e_{i j}^{n}\right)^{2}+\left(e_{i, j-1}^{n}\right)^{2}\right]+\frac{\mu^{2} k}{4 a}\left[\left(e_{i, j+1}^{n}\right)^{2}+\left(e_{i, j-1}^{n}\right)^{2}\right]+k^{2} C^{2}\left(e_{i j}^{n}\right)^{2}\right]+\frac{a k}{4}\left[\left(\delta_{y} e_{i, j+\frac{1}{2}}^{n}\right)^{2}\right. \\
& \left.+\left(\delta_{y} e_{i, j-\frac{1}{2}}^{n}\right)^{2}\right]+\frac{\mu^{2} k}{16 a}\left[\left(e_{i, j+1}^{n}\right)^{2}+\left(e_{i, j-1}^{n}\right)^{2}\right]+\frac{C^{2} k^{2}}{4}\left(e_{i j}^{n}\right)^{2}+\frac{a k}{4}\left[\left(\delta_{y} e_{i, j+\frac{1}{2}}^{n}\right)^{2}+\left(\delta_{y} e_{i, j-\frac{1}{2}}^{n}\right)^{2}\right] \\
& \left.+\frac{\mu^{2} k}{16 a}\left[\left(e_{i, j+1}^{n}\right)^{2}+\left(e_{i, j-1}^{n}\right)^{2}\right]+\frac{C^{2} k^{2}}{4}\left(e_{i j}^{n}\right)^{2}+\frac{3 k}{8}\left[\left(e_{i, j+1}^{n}\right)^{2}+4\left(e_{i j}^{n}\right)^{2}+\left(e_{i, j-1}^{n}\right)^{2}\right]+\frac{C^{2} k}{8}\left(e_{i j}^{n}\right)^{2}\right\} .
\end{aligned}
$$

Summing estimate (4.3) up from $i, j=1,2, \ldots, M-1$, and rearranging of terms we get

$$
\begin{aligned}
\sum_{i, j=1}^{M-1}\left(e_{i j}^{*}\right)^{2} \leqslant & \sum_{i, j=1}^{M-1}\left(e_{i j}^{n}\right)^{2}+\frac{a k}{h^{2}} \sum_{i, j=1}^{M-1}\left(e_{i, j+1}^{n}-2 e_{i j}^{n}+e_{i, j-1}^{n}\right) e_{i j}^{n}-\frac{\mu k}{2 h} \sum_{i, j=1}^{M-1}\left(e_{i, j+1}^{n}-e_{i, j-1}^{n}\right) e_{i j}^{n} \\
& +\frac{a k}{2} \sum_{i, j=1}^{M-1}\left[\left(\delta_{y} e_{i, j+\frac{1}{2}}^{n}\right)^{2}+\left(\delta_{y} e_{i, j-\frac{1}{2}}^{n}\right)^{2}\right]+\frac{3 k}{2} \sum_{i, j=1}^{M-1}\left[\left(e_{i, j+1}^{n}\right)^{2}+4\left(e_{i j}^{n}\right)^{2}+\left(e_{i, j-1}^{n}\right)^{2}\right] \\
& +\frac{\mu^{2} k}{8 a}(1+3 k) \sum_{i, j=1}^{M-1}\left[\left(e_{i, j+1}^{n}\right)^{2}+\left(e_{i, j-1}^{n}\right)^{2}\right]+k\left(C+\frac{5}{4}+\frac{C^{2}}{8}+\frac{C^{2} k}{2}+\frac{3 C^{2} k^{2}}{2}\right) \sum_{i, j=1}^{M-1}\left(e_{i j}^{n}\right)^{2} \\
& +\frac{9 \widehat{C}_{1}^{2} k^{3}}{2}(M-1)^{2}\left(\frac{5}{2}+k\right)\left[1+\widehat{C}_{2} h^{2}+\widehat{C}_{3} h^{4}\right]^{2}+4 C_{r}^{2} k(M-1)^{2}\left(k^{2}+k^{4}+k^{5}+h^{4}+h^{8}\right) .
\end{aligned}
$$


From the boundary condition (2.32), $e_{i M}^{n}=e_{i 0}^{n}=0$, for $i=0,1, \ldots, M$. Using this, it is not difficult to observe that

$$
\begin{aligned}
\sum_{i, j=1}^{M-1}\left[\left(\delta_{y} e_{i, j+\frac{1}{2}}^{n}\right)^{2}+\left(\delta_{y} e_{i, j-\frac{1}{2}}^{n}\right)^{2}\right] & =\sum_{i, j=1}^{M-1}\left(\delta_{y} e_{i, j+\frac{1}{2}}^{n}\right)^{2}+\sum_{i, j=1}^{M-1}\left(\delta_{y} e_{i, j-\frac{1}{2}}^{n}\right)^{2} \\
& \leqslant \sum_{i=1}^{M-1} \sum_{j=0}^{M-1}\left(\delta_{y} e_{i, j+\frac{1}{2}}^{n}\right)^{2}+\sum_{i=1}^{M-1} \sum_{j=0}^{M-1}\left(\delta_{y} e_{i, j+\frac{1}{2}}^{n}\right)^{2}=2 \sum_{i=1}^{M-1} \sum_{j=0}^{M-1}\left(\delta_{y} e_{i, j+\frac{1}{2}}^{n}\right)^{2} .
\end{aligned}
$$

Furthermore, using inequality $\left(1+\widehat{\mathrm{C}}_{2} \mathrm{~h}^{2}+\widehat{\mathrm{C}}_{3} \mathrm{~h}^{4}\right)^{2} \leqslant 3\left(1+\widehat{\mathrm{C}}_{2}^{2} \mathrm{~h}^{4}+\widehat{\mathrm{C}}_{3}^{2} \mathrm{~h}^{8}\right)$, Lemmas 2.1 and 4.3 , multiplying both sides of inequality (4.4) by $h^{2}$ and performing simple calculations, we obtain

$$
\begin{aligned}
h^{2} \sum_{i, j=1}^{M-1}\left(e_{i j}^{*}\right)^{2} \leqslant & h^{2} \sum_{i, j=1}^{M-1}\left(e_{i j}^{n}\right)^{2}-a k\left\|\delta_{y} e^{n}\right\|_{L^{2}(\Omega)}^{2}+a k\left\|\delta_{y} e^{n}\right\|_{L^{2}(\Omega)}^{2}+4 C_{r}^{2} k(M-1)^{2} h^{2}\left(k^{2}+k^{4}+k^{5}+h^{4}+h^{8}\right) \\
& +k\left[\frac{41}{4}+\frac{\mu^{2}}{8 a}+C+\frac{C^{2}}{8}+\frac{3 \mu^{2} k}{8 a}+\frac{C^{2} k}{2}+\frac{3 C^{2} k^{2}}{2}\right] h^{2} \sum_{i, j=1}^{M-1}\left(e_{i j}^{n}\right)^{2} \\
& +\frac{27 \widehat{C}_{1}^{2} k^{3}}{2}(M-1)^{2} h^{2}\left(\frac{5}{2}+k\right)\left[1+\widehat{C}_{2}^{2} h^{4}+\widehat{C}_{3}^{2} h^{8}\right] \\
\leqslant & h^{2} \sum_{i, j=1}^{M-1}\left(e_{i j}^{n}\right)^{2}+k\left[\frac{41}{4}+\frac{\mu^{2}}{8 a}+C+\frac{C^{2}}{8}+\frac{3 \mu^{2} k}{8 a}+\frac{C^{2} k}{2}+\frac{3 C^{2} k^{2}}{2}\right] h^{2} \sum_{i, j=1}^{M-1}\left(e_{i j}^{n}\right)^{2} \\
& +4 C_{r}^{2} k\left(k^{2}+k^{4}+k^{5}+h^{4}+h^{8}\right)+\frac{27 \widehat{C}_{1}^{2} k^{3}}{2}\left(\frac{5}{2}+k\right)\left[1+\widehat{C}_{2}^{2} h^{4}+\widehat{C}_{3}^{2} h^{8}\right]
\end{aligned}
$$

which is implies

$$
\left\|e^{*}\right\|_{L^{2}(\Omega)}^{2} \leqslant\left\|e^{\mathfrak{n}}\right\|_{L^{2}(\Omega)}^{2}+\widehat{C}_{4} k\left\{\left(1+k+k^{2}\right)\left\|e^{\mathfrak{n}}\right\|_{L^{2}(\Omega)}^{2}+k^{2}(1+k)\left(1+h^{4}+h^{8}\right)+k^{2}+k^{4}+k^{5}+h^{4}+h^{8}\right\},
$$

where we absorbed all the constants into a constant $\widehat{C}_{4}>0$.

Analogously, it is not hard to show that

$$
\left\|e^{* *}\right\|_{\mathrm{L}^{2}(\Omega)}^{2} \leqslant\left\|e^{*}\right\|_{\mathrm{L}^{2}(\Omega)}^{2}+\widehat{\mathrm{C}}_{5} \mathrm{k}\left\{\left(1+\mathrm{k}+\mathrm{k}^{2}\right)\left\|\mathrm{e}^{*}\right\|_{\mathrm{L}^{2}(\Omega)}^{2}+\mathrm{k}^{2}(1+\mathrm{k})\left(1+\mathrm{h}^{4}+\mathrm{h}^{8}\right)+\mathrm{k}^{2}+\mathrm{k}^{4}+\mathrm{k}^{5}+\mathrm{h}^{4}+\mathrm{h}^{8}\right\},
$$

where all the constants have been absorbed into a positive constant $\widehat{C}_{5}$, and

$$
\left\|e^{n+1}\right\|_{\mathrm{L}^{2}(\Omega)}^{2} \leqslant\left\|e^{* *}\right\|_{\mathrm{L}^{2}(\Omega)}^{2}+\widehat{\mathrm{C}}_{6} \mathrm{k}\left\{\left(1+k+\mathrm{k}^{2}\right)\left\|e^{* *}\right\|_{\mathrm{L}^{2}(\Omega)}^{2}+\mathrm{k}^{2}(1+\mathrm{k})\left(1+\mathrm{h}^{4}+\mathrm{h}^{8}\right)+\mathrm{k}^{2}+\mathrm{k}^{4}+\mathrm{k}^{5}+\mathrm{h}^{4}+\mathrm{h}^{8}\right\},
$$

where we absorbed all the constants into a constant $\widehat{\mathrm{C}}_{6}>0$.

For the convenient of writing, we should set

$$
\theta_{1}(k, h)=1+k+k^{2} \text {, and } \theta_{2}(k, h)=k^{2}(1+k)\left(1+h^{4}+h^{8}\right)+k^{2}+k^{4}+k^{5}+h^{4}+h^{8} .
$$

Combining estimates (4.5)-(4.8), straightforward calculations result in

$$
\begin{aligned}
\left\|e^{n+1}\right\|_{L^{2}(\Omega)}^{2} \leqslant & \left\|e^{n}\right\|_{L^{2}(\Omega)}^{2}+k\left\{\widehat{C}_{4}+\widehat{C}_{5}+\widehat{C}_{6}+k\left[\widehat{C}_{4} \widehat{C}_{5}+\widehat{C}_{6}\left(\widehat{C}_{4}+\widehat{C}_{5}\right)+k \widehat{C}_{4} \widehat{C}_{5} \widehat{C}_{6} \theta_{1}(k, h)\right] \theta_{1}(k, h)\right\} \\
& +\theta_{1}(k, h)\left\|e^{n}\right\|_{L^{2}(\Omega)}^{2} \\
& +k\left\{\widehat{C}_{4}+\widehat{C}_{5}+\widehat{C}_{6}+k\left[\widehat{C}_{4} \widehat{C}_{5}+\widehat{C}_{4} \widehat{C}_{6}+\widehat{C}_{5} \widehat{C}_{6}+k \widehat{C}_{4} \widehat{C}_{5} \widehat{C}_{6} \theta_{1}(k, h)\right] \theta_{1}(k, h)\right\} \theta_{2}(k, h) .
\end{aligned}
$$


Absorbing all the constants into a positive constant $\widehat{C}_{7}$, we get

$$
\begin{aligned}
\left\|e^{n+1}\right\|_{L^{2}(\Omega)}^{2} \leqslant & \left\|e^{\mathfrak{n}}\right\|_{L^{2}(\Omega)}^{2}+\widehat{C}_{7} k\left\{\left[1+k\left(1+k \theta_{1}(k, h)\right) \theta_{1}(k, h)\right] \theta_{1}(k, h)\left\|e^{n}\right\|_{L^{2}(\Omega)}^{2}\right. \\
& \left.+\left[1+k\left[1+k \theta_{1}(k, h)\right] \theta_{1}(k, h)\right] \theta_{2}(k, h)\right\} .
\end{aligned}
$$

Summing this up from $n=0,1,2, \ldots, p-1$, for any positive integer $p$ such that $1 \leqslant p \leqslant N$, it is easy to see that

$$
\begin{aligned}
\left\|e^{p}\right\|_{L^{2}(\Omega)}^{2} \leqslant & \left\|e^{0}\right\|_{L^{2}(\Omega)}^{2}+\widehat{C}_{7 k}\left\{\left[1+k\left(1+k \theta_{1}(k, h)\right) \theta_{1}(k, h)\right] \theta_{1}(k, h) \sum_{n=0}^{p-1}\left\|e^{n}\right\|_{L^{2}(\Omega)}^{2}\right. \\
& \left.+p\left[1+k\left[1+k \theta_{1}(k, h)\right] \theta_{1}(k, h)\right] \theta_{2}(k, h)\right\} .
\end{aligned}
$$

It follows from the initial condition defined in (2.32), that $e_{i j}^{0}=0$, for $0 \leqslant i, j \leqslant M$. The application of the Gronwall Lemma to estimate (4.9) yields

$$
\left\|e^{\mathfrak{p}}\right\|_{L^{2}(\Omega)}^{2} \leqslant \widehat{C}_{7} p k \exp \left\{\widehat{C}_{7} p k\left[1+k\left(1+k \theta_{1}(k, h)\right) \theta_{1}(k, h)\right] \theta_{1}(k, h)\right\}\left[1+k\left[1+k \theta_{1}(k, h)\right] \theta_{1}(k, h)\right] \theta_{2}(k, h) .
$$

But $p \leqslant N$ and $k=\frac{T}{N}$, so $\widehat{C}_{7} k p \leqslant \widehat{C}_{7} T$. This fact combining with inequality (4.10) gives

$$
\left\|e^{\mathfrak{p}}\right\|_{\mathrm{L}^{2}(\Omega)}^{2} \leqslant \widehat{C}_{7} \mathrm{~T} \exp \left\{\widehat{\mathcal{C}}_{7} \mathrm{~T}\left[1+\mathrm{k}\left(1+\mathrm{k} \theta_{1}(k, h)\right) \theta_{1}(k, h)\right] \theta_{1}(k, h)\right\}\left[1+k\left[1+k \theta_{1}(k, h)\right] \theta_{1}(k, h)\right] \theta_{2}(k, h)^{2},
$$

The square root of this provides

$$
\begin{aligned}
\left\|e^{\mathfrak{p}}\right\|_{L^{2}(\Omega)} \leqslant & \sqrt{\widehat{C}_{7} T\left[1+k\left[1+k \theta_{1}(k, h)\right] \theta_{1}(k, h)\right]} \\
& \times \exp \left\{\frac{\widehat{C}_{7} T}{2}\left[1+k\left(1+k \theta_{1}(k, h)\right) \theta_{1}(k, h)\right] \theta_{1}(k, h)\right\} \sqrt{\theta_{2}(k, h)} .
\end{aligned}
$$

Now, it comes from relation (4.8) that

$$
\theta_{2}(k, h)=k^{2}(1+k)\left(1+h^{4}+h^{8}\right)+k^{2}+k^{4}+k^{5}+h^{4}+h^{8} \leqslant\left(k+h^{4}\right)^{2} \psi(k, h),
$$

where $\psi(k, h)=1+k^{2}+k^{3}+h^{2}+(1+k)\left(1+h^{4}+h^{8}\right)$. Furthermore, $1+k\left[1+k \theta_{1}(k, h)\right] \theta_{1}(k, h)=1+$ $k\left[1+\left(1+k+k^{2}\right)\right]\left(1+k+k^{2}\right) \leqslant\left(1+k+k^{2}\right)^{4}$ and

$\left[1+k\left(1+k \theta_{1}(k, h)\right) \theta_{1}(k, h)\right] \theta_{1}(k, h)=\left[1+k\left(1+k\left(1+k+k^{2}\right)\right)\left(1+k+k^{2}\right)\right]\left(1+k+k^{2}\right) \leqslant\left(1+k+k^{2}\right)^{5}$.

Finally, taking the maximum over $p$ of estimate (4.11), for $0 \leqslant p \leqslant N$, and applying equation (4.1), this ends the proof of Theorem 4.1.

\section{Numerical experiments and convergence rate}

In this section we construct exact solutions to the two-dimensional time-dependent nonlinear advectiondiffusion equation with constant coefficients (1.1)-(1.3). Furthermore, we consider another example described in the literature [16] to demonstrate the efficiency and effectiveness of the proposed numerical scheme in two-dimensional case. In each case we obtain satisfactory results, so our algorithm performances provide good results for multidimensional problems. The predicted convergence rate from the theoretical result is confirmed (see Section 2, page 8, paragraph below equation (2.28), lines 3-4). The convergence rate is obtained by listing in Tables $1-6$, the errors between the computed solution and the 
analytical one with different values of mesh size $h$ and time step $k$, satisfying $k=\frac{1}{2} h^{2}$. Finally, we look at the error estimates of our proposed method for the parameter $T=1$ and $a=\mu=1$.

In order to construct analytical solutions to the initial-boundary value problem (1.1)-(1.3), we should assume that they can be expressed as $\bar{\phi}(x, y, t)=[1+\exp (c t+d x+b y)]^{\alpha}$, where $\alpha$ is a real number. By simple calculations, it is not difficult to observe that

$$
\begin{aligned}
& \bar{\phi}_{t}(x, y, t)=\alpha c \exp (c t+d x+b y)[1+\exp (c t+d x+b y)]^{\alpha-1}, \\
& \bar{\phi}_{x}(x, y, t)=\alpha d \exp (c t+d x+b y)[1+\exp (c t+d x+b y)]^{\alpha-1}, \\
& \bar{\phi}_{y}(x, y, t)=\alpha b \exp (c t+d x+b y)[1+\exp (c t+d x+b y)]^{\alpha-1},
\end{aligned}
$$

and

$$
\bar{\phi}_{x x}(x, y, t)=\alpha d^{2} \exp (c t+d x+b y)[1+\alpha \exp (c t+d x+b y)][1+\exp (c t+d x+b y)]^{\alpha-2} .
$$

In a similar manner

$$
\bar{\phi}_{y y}(x, y, t)=\alpha b^{2} \exp (c t+d x+b y)[1+\alpha \exp (c t+d x+b y)][1+\exp (c t+d x+b y)]^{\alpha-2} .
$$

Combining equations (5.1)-(5.5), it is not hard to see that

$$
\begin{aligned}
\bar{\phi}_{t}+\bar{\phi}_{x}+\bar{\phi}_{y}-\left(\bar{\phi}_{x x}+\bar{\phi}_{y y}\right)= & \alpha \exp (c t+d x+b y)(1+\exp (c t+d x+b y))^{\alpha-1}\left\{c+b+d-\left(b^{2}+d^{2}\right)\right. \\
& \left.\times[1+\alpha \exp (c t+d x+b y)](1+\exp (c t+d x+b y))^{-1}\right\} .
\end{aligned}
$$

Setting $c+b+d=-\left(d^{2}+b^{2}\right)$, it holds

$$
\begin{aligned}
\bar{\phi}_{t}+\bar{\phi}_{x}+\bar{\phi}_{y}-\left(\bar{\phi}_{x x}+\bar{\phi}_{y y}\right)= & -\alpha\left(d^{2}+b^{2}\right) \exp (c t+d x+b y)(1+\exp (c t+d x+b y))^{\alpha-1} \\
& \times\left\{1+[1+\alpha \exp (c t+d x+b y)][1+\exp (c t+d x+b y)]^{-1}\right\} .
\end{aligned}
$$

For $\alpha=1$, equation (5.6) becomes

$$
\bar{\phi}_{\mathrm{t}}+\bar{\phi}_{x}+\bar{\phi}_{y}-\left(\bar{\phi}_{x x}+\bar{\phi}_{y y}\right)=-2\left(d^{2}+b^{2}\right) \exp (c t+d x+b y) .
$$

Assuming $b \neq 0$ and $d^{2}+b^{2}=1$, this is equivalent to $b^{2}=1-d^{2}$. Since $b^{2}>0$, this implies $d^{2}<1$. We can take $d=-\frac{\sqrt{2}}{2}$ and $b=-\frac{\sqrt{2}}{2}$. Thus $c=-1+\sqrt{2}$. The analytical solution is given by $\bar{\phi}(x, y, t)=$ $1+\exp \left((-1+\sqrt{2}) t-\frac{\sqrt{2}}{2} x-\frac{\sqrt{2}}{2} y\right)$, for every $t \in[0,1],(x, y) \in[0,1]^{2}$ and the function $f$ is given by $f(\bar{\phi})=2(1-\bar{\phi})$. The initial and boundary conditions are determined by the exact solution.

Now, if $\alpha=-1$, performing direct computations, equation (5.6) yields

$$
\bar{\phi}_{t}+\bar{\phi}_{x}+\bar{\phi}_{y}-\left(\bar{\phi}_{x x}+\bar{\phi}_{y y}\right)=2\left(d^{2}+b^{2}\right) \exp (c t+d x+b y)[1+\exp (c t+d x+b y)]^{-3} .
$$

For $b \neq 0$ and $d^{2}+b^{2}=1$, the values $d=-\frac{\sqrt{3}}{2}$ and $b=-\frac{1}{2}$ satisfy this equation. It comes from equation $c+b+d=-\left(d^{2}+b^{2}\right)$ that $c=-1+\frac{\sqrt{3}+1}{2}$. Thus, the exact solution is given by $\bar{\phi}(x, y, t)=$ $\left[1+\exp \left(\left(-1+\frac{\sqrt{3}+1}{2}\right) t-\frac{\sqrt{3}}{2} x-\frac{1}{2} y\right)\right]^{-1}$, for any $t \in[0,1],(x, y) \in[0,1]^{2}$ and the function $f$ is defined as $f(\bar{\phi})=2(1-\bar{\phi}) \bar{\phi}^{2}$. The initial and boundary conditions are given by this solution.

In this analysis, we assume that the mesh size $h \in\left\{\frac{1}{2^{l}}, l=1,2, \ldots, 5\right\}$ and time step $k=2^{-l}$, $l=1,2, \ldots, 11$. In addition, we set $\mathrm{k}=\frac{1}{2} \mathrm{~h}^{2}$ and we compute the error estimates: $\|\mid \mathrm{E}(\phi)\| \|_{\mathrm{L}^{2}\left(0, \mathrm{~T} ; \mathrm{L}^{2}\right)}$, $\|\mathrm{E}(\phi) \mid\|_{\mathrm{L}^{\infty}\left(0, \mathrm{~T} ; \mathrm{L}^{2}\right)}$ and $\||\mathrm{E}(\phi)|\|_{\mathrm{L}^{1}\left(0, \mathrm{~T} ; \mathrm{L}^{2}\right)}$ related to the three-level time-split method to see that the algorithm is stable, second order convergent in time and fourth order accurate in space. Furthermore, we plot the $\mathrm{L}^{2}$-norm of the analytic solution, the numerical one and the errors versus $n$. From this study, we 
observe that the three-level time-split MacCormack method is efficient and effective than a broad range of numerical schemes widely studied in the literature. Finally, when $h$ varies in the given range, it comes from Tables 1-6 that the error terms $O\left(k^{\beta}\right)+O\left(h^{\theta}\right)$ are dominated by the h-terms $O\left(h^{\theta}\right)$ (or k-terms $\mathrm{O}\left(\mathrm{k}^{\beta}\right)$ ). So, the ratio $\mathrm{r}_{\phi}^{\mathrm{m}}$, where $\mathrm{m}=1,2, \infty$, of the approximate errors on two adjacent mesh levels $\Omega_{2 h}$ and $\Omega_{h}$ is approximately $(2 h)^{\theta} / h^{\theta}=2^{\theta}$, where $m$ refers to the $L^{m}\left(0, T ; L^{2}(\Omega)\right.$-error norm. Thus, we can use $r_{\phi}^{m}$ to estimate the corresponding convergence rate with respect to $h$. Define the norms for the approximate solution $\phi$, the exact one $\bar{\phi}$, and the errors $E(\phi)$, as follows

$$
\begin{aligned}
\||\phi|\|_{\mathrm{L}^{2}\left(0, \mathrm{~T} ; \mathrm{L}^{2}\right)} & =\left[k \sum_{n=0}^{N}\left\|\phi^{\mathrm{n}}\right\|_{\mathrm{L}_{\mathrm{f}}^{2}}^{2}\right]^{\frac{1}{2}}, & \|\bar{\phi} \mid\|_{\mathrm{L}^{2}\left(0, \mathrm{~T} ; \mathrm{L}^{2}\right)}=\left[k \sum_{n=0}^{N}\left\|\bar{\phi}^{n}\right\|_{\mathrm{L}_{\mathrm{f}}^{2}}^{2}\right]^{\frac{1}{2}}, \\
\||\mathrm{E}(\phi)|\|_{\mathrm{L}^{2}\left(0, \mathrm{~T} ; \mathrm{L}^{2}\right)} & =\left[k \sum_{\mathrm{n}=0}^{\mathrm{N}}\left\|\phi^{\mathrm{n}}-\bar{\phi}^{\mathrm{n}}\right\|_{\mathrm{L}_{\mathrm{f}}^{2}}^{2}\right]^{\frac{1}{2}}, & \|\mathrm{E}(\phi) \mid\|_{\mathrm{L}^{1}\left(0, \mathrm{~T} ; \mathrm{L}^{2}\right)}=k \sum_{n=0}^{N}\left\|\phi^{n}-\bar{\phi}^{n}\right\|_{\mathrm{L}_{\mathrm{f}}^{2}}
\end{aligned}
$$

and

$$
\||E(\phi)|\|_{L^{\infty}\left(0, T ; L^{2}\right)}=\max _{0 \leqslant n \leqslant N}\left\|\phi^{n}-\bar{\phi}^{n}\right\|_{L_{f}^{2}} .
$$

Test 5.1. Let $\Omega$ be the unit square $(0,1) \times(0,1)$ and $\mathrm{T}$ be the final time, $\mathrm{T}=1$. We assume that the parameters $a=\mu=1$, in such a way that the exact solution $\bar{\phi}$ and the function $f$ are given by

$$
\bar{\phi}(x, y, t)=1+\exp \left((-1+\sqrt{2}) t-\frac{\sqrt{2}}{2} x-\frac{\sqrt{2}}{2} y\right), \text { and } f(\bar{\phi})=2(1-\bar{\phi}) .
$$

The initial and boundary conditions are given by this solution. We recall that the mesh size and time step: $h \in\left\{\frac{1}{2}, \frac{1}{2^{2}}, \frac{1}{2^{3}}, \frac{1}{2^{4}}, \frac{1}{2^{5}}\right\}$ and $k \in\left\{\frac{1}{2^{2}}, \frac{1}{2^{3}}, \frac{1}{2^{4}}, \frac{1}{2^{5}}, \frac{1}{2^{6}}, \frac{1}{2^{7}}, \frac{1}{2^{8}}, \frac{1}{2^{9}}, \frac{1}{2^{10}}, \frac{1}{2^{11}}\right\}$.

Table 1: Convergence rate $\mathrm{O}\left(\mathrm{h}^{\theta}+\Delta \mathrm{t}^{\beta}\right)$ for time-split MacCormack by $r_{\phi}^{m}$, under the time step restriction $(2.33)$, i.e., $\max \left\{\frac{2 k}{h^{2}}, \frac{k}{h}\right\} \leqslant$ 1 , with varying time step $\mathrm{k}=\Delta \mathrm{t}$ and mesh grid $\mathrm{h}=\Delta \mathrm{x}=\Delta \mathrm{y}$ and $\mathrm{k}=\frac{1}{2} \mathrm{~h}^{2}$.

\begin{tabular}{|c|c|c|c|c|c|c|}
\hline $\mathrm{h}$ & $\|\mathrm{E}(\phi) \mid\|_{\mathrm{L}^{2}}$ & $\mathrm{r}_{\phi}^{2}$ & $\|\mathrm{E}(\phi)\| \|_{\mathrm{L}^{\infty}}$ & $\mathrm{r}_{\phi}^{\infty}$ & $\|\mathrm{E}(\phi)\|_{\mathrm{L}^{1}}$ & $\mathrm{r}_{\phi}^{1}$ \\
\hline $2^{-1}$ & $0.2720 \times 10^{-1}$ & - & $0.3240 \times 10^{-1}$ & - & $0.2700 \times 10^{-1}$ & - \\
\hline $2^{-2}$ & $0.6200 \times 10^{-2}$ & 4.3871 & $0.7500 \times 10^{-2}$ & 4.3200 & $0.6100 \times 10^{-2}$ & 4.4262 \\
\hline $2^{-3}$ & $0.1600 \times 10^{-2}$ & 3.8750 & $0.2000 \times 10^{-2}$ & 3.7500 & $0.1600 \times 10^{-2}$ & 3.8125 \\
\hline $2^{-4}$ & $0.4000 \times 10^{-3}$ & 4.0000 & $0.5000 \times 10^{-3}$ & 4.0000 & $0.4000 \times 10^{-3}$ & 4.0000 \\
\hline $2^{-5}$ & $0.1062 \times 10^{-3}$ & 3.7665 & $0.1302 \times 10^{-3}$ & 3.8402 & $0.1049 \times 10^{-3}$ & 3.8132 \\
\hline
\end{tabular}

Table 2: Convergence rate $\mathrm{O}\left(\mathrm{h}^{\theta}+\Delta \mathrm{t}^{\beta}\right)$ for time-split MacCormack by $r_{\phi}^{m}$, under the time step restriction $(2.33)$, i.e., $\max \left\{\frac{2 \mathrm{k}}{\mathrm{h}^{2}}, \frac{\mathrm{k}}{\mathrm{h}}\right\} \leqslant$ 1 , with varying time step $\mathrm{k}=\Delta \mathrm{t}$ and mesh grid $\mathrm{h}=\Delta \mathrm{x}=\Delta \mathrm{y}$ and $\mathrm{k}=\mathrm{h}^{2}$.

\begin{tabular}{|c|c|c|c|c|c|c|}
\hline $\mathrm{h}$ & $\||\mathrm{E}(\phi)|\|_{\mathrm{L}^{2}}$ & $\mathrm{r}_{\phi}^{2}$ & $\|\mathrm{E}(\phi) \mid\|_{\mathrm{L}^{\infty}}$ & $\mathrm{r}_{\phi}^{\infty}$ & $\|\mathrm{E}(\phi)\|_{\mathrm{L}^{1}}$ & $\mathrm{r}_{\phi}^{1}$ \\
\hline $2^{-1}$ & 0.1109 & - & 0.1292 & - & 0.1095 & - \\
\hline $2^{-2}$ & $0.7120 \times 10^{-1}$ & 1.5576 & $0.1912 \times 10^{0}$ & 0.6757 & $0.5130 \times 10^{-1}$ & 2.1345 \\
\hline $2^{-3}$ & $0.3793 \times 10^{20}$ & $0.1877 \times 10^{-20}$ & $2.7815 \times 10^{20}$ & $0.6874 \times 10^{-21}$ & $0.7240 \times 10^{19}$ & $0.7086 \times 10^{-20}$ \\
\hline
\end{tabular}

Test 5.2. Now, let $\Omega$ be the unit square $(0,1)^{2}$ and $T=1$. The diffusive term $a$ is assumed equals 1 and the parameter $\mu=1$. The analytic solution $\bar{\phi}$ together with the function $f$ are defined as

$$
\bar{\phi}(x, y, t)=\left[1+\exp \left(\left(-1+\frac{\sqrt{3}+1}{2}\right) t-\frac{\sqrt{3}}{2} x-\frac{1}{2} y\right)\right]^{-1} \text { and } f(\bar{\phi})=2(1-\bar{\phi}) \bar{\phi}^{2} \text {. }
$$


The initial and boundary conditions also are given by the exact solution $\bar{\phi}$. Similar to Test 5.1 we take both mesh size and time step: $h \in\left\{\frac{1}{2^{2}}, \frac{1}{2^{2}}, \frac{1}{2^{3}}, \frac{1}{2^{4}}, \frac{1}{2^{5}}\right\}$ and $k \in\left\{\frac{1}{2^{2}}, \frac{1}{2^{3}}, \frac{1}{2^{4}}, \frac{1}{2^{5}}, \frac{1}{2^{6}}, \frac{1}{2^{7}}, \frac{1}{2^{8}}, \frac{1}{2^{9}}, \frac{1}{2^{10}}, \frac{1}{2^{11}}\right\}$.

Table 3: Analysis of convergence rates $\mathrm{O}\left(h^{\theta}+\Delta t^{\beta}\right)$ for the three-level time-split MacCormack by $r_{\phi}^{m}$, with varying spacing $h$ and time step $k$, under the time step restriction (2.33), that is, $\max \left\{\frac{2 k}{h^{2}}, \frac{k}{h}\right\} \leqslant 1$ and $k=\frac{1}{2} h^{2}$.

\begin{tabular}{|c|c|c|c|c|c|c|}
\hline $\mathrm{h}$ & $\|\mathrm{IE}(\phi) \mid\|_{\mathrm{L}^{2}}$ & $\mathrm{r}_{\phi}^{2}$ & $\|\mathrm{E}(\phi) \mid\|_{\mathrm{L}^{\infty}}$ & $\mathrm{r}_{\phi}^{\infty}$ & $\|\mathrm{E}(\phi) \mid\|_{\mathrm{L}^{1}}$ & $\mathrm{r}_{\phi}^{1}$ \\
\hline $2^{-1}$ & $0.9100 \times 10^{-2}$ & - & $0.1680 \times 10^{-1}$ & - & $0.8500 \times 10^{-2}$ & - \\
\hline $2^{-2}$ & $0.2100 \times 10^{-2}$ & 4.3333 & $0.4400 \times 10^{-2}$ & 3.8182 & $0.21 \times 10^{-2}$ & 4.0476 \\
\hline $2^{-3}$ & $0.5000 \times 10^{-3}$ & 4.2000 & $0.1100 \times 10^{-2}$ & 4.0000 & $0.5000 \times 10^{-3}$ & 4.2000 \\
\hline $2^{-4}$ & $0.1390 \times 10^{-3}$ & 3.5971 & $0.2820 \times 10^{-3}$ & 3.9007 & $0.1378 \times 10^{-3}$ & 3.6284 \\
\hline $2^{-5}$ & $0.3520 \times 10^{-4}$ & 3.9448 & $0.7050 \times 10^{-4}$ & 4.0000 & $0.3490 \times 10^{-4}$ & 3.9484 \\
\hline
\end{tabular}

Table 4: Analysis of convergence rates $\mathrm{O}\left(h^{\theta}+\Delta t^{\beta}\right)$ for the three-level time-split MacCormack by $r_{\phi}^{m}$, with varying spacing $h$ and time step $k$, under the time step restriction (2.33), that is, $\max \left\{\frac{2 k}{h^{2}}, \frac{k}{h}\right\} \leqslant 1$ and $k=h^{2}$.

\begin{tabular}{|c|c|c|c|c|c|c|}
\hline $\mathrm{h}$ & $\|\mathrm{E}(\phi) \mid\|_{\mathrm{L}^{2}}$ & $\mathrm{r}_{\phi}^{2}$ & $\|\mathrm{E}(\phi)\|_{\mathrm{L}^{\infty}}$ & $\mathrm{r}_{\phi}^{\infty}$ & $\|\mathrm{E}(\phi)\|_{\mathrm{L}^{1}}$ & $\mathrm{r}_{\phi}^{1}$ \\
\hline $2^{-1}$ & $0.3350 \times 10^{-1}$ & - & $0.4840 \times 10^{-1}$ & - & $0.318 \times 10^{-1}$ & - \\
\hline $2^{-2}$ & $0.1110 \times 10^{-1}$ & 3.0180 & $0.2000 \times 10^{-1}$ & 2.4200 & $0.1030 \times 10^{-1}$ & 3.0874 \\
\hline $2^{-3}$ & $\mathrm{NaN} \times 10^{251}$ & - & $\mathrm{Inf} \times 10^{251}$ & - & $\mathrm{NaN} \times 10^{251}$ & - \\
\hline
\end{tabular}

Test 5.3. Finally, let $\Omega$ be the unit square $(0,1) \times(0,1)$ and $T=1$. Assuming that $a=\mu=1$, the exact solution $\bar{\phi}$ is given in [16] by

$$
\bar{\phi}(x, y, t)=\exp (-t) x y(1-x)(1-y) .
$$

The function $f(\bar{\phi})=-\bar{\phi}+\exp (-t)[x(1-x)(3-2 y)+y(1-y)(3-2 x)]$, the initial and boundary conditions are given by the exact solution $\bar{\phi}$.

Similar to both Tests 5.1 and 5.2 both time step and grid spacing are chosen such that: $k \in\left\{\frac{1}{2^{2}}, \frac{1}{2^{3}}, \frac{1}{2^{4}}, \frac{1}{2^{5}}\right.$, $\left.\frac{1}{2^{6}}, \frac{1}{2^{7}}, \frac{1}{2^{8}}, \frac{1}{2^{9}}, \frac{1}{2^{10}}, \frac{1}{2^{11}}\right\}$ and $h \in\left\{\frac{1}{2}, \frac{1}{2^{2}}, \frac{1}{2^{3}}, \frac{1}{2^{4}}, \frac{1}{2^{5}}\right\}$. We compute the error estimates: $E(\phi)$ related to a three-level time-split MacCormack approach to see that the method is second order convergent in time and fourth order accurate in space. Furthermore, we plot the errors together with the energies versus $n$ to see the efficiency and effectiveness of the considered algorithm.

Table 5: Convergence rates $\mathrm{O}\left(\mathrm{h}^{\theta}+\Delta \mathrm{t}^{\beta}\right)$ for time-split MacCormack by $r_{\mathfrak{u}}^{\mathrm{m}}$, with varying spacing $\mathrm{h}$ and time step $\Delta t$ and $k=\frac{1}{2} h^{2}$.

\begin{tabular}{|c|c|c|c|c|c|c|}
\hline $\mathrm{h}$ & $\||\mathrm{E}(\phi)|\|_{\mathrm{L}^{2}}$ & $\mathrm{r}_{\phi}^{2}$ & $\|\mathrm{E}(\phi) \mid\|_{\mathrm{L}^{\infty}}$ & $\mathrm{r}_{\phi}^{\infty}$ & $\|\mathrm{E}(\phi) \mid\|_{\mathrm{L}^{1}}$ & $\mathrm{r}_{\phi}^{1}$ \\
\hline $2^{-1}$ & $0.7900 \times 10^{-2}$ & - & $0.1140 \times 10^{-1}$ & - & $0.7600 \times 10^{-2}$ & - \\
\hline $2^{-2}$ & $0.1400 \times 10^{-2}$ & 5.6429 & $0.2000 \times 10^{-2}$ & 5.7000 & $0.1400 \times 10^{-2}$ & 5.4286 \\
\hline $2^{-3}$ & $0.3257 \times 10^{-3}$ & 4.2984 & $0.4412 \times 10^{-3}$ & 4.5331 & $0.3155 \times 10^{-3}$ & 4.4374 \\
\hline $2^{-4}$ & $0.7920 \times 10^{-4}$ & 4.1124 & $0.1072 \times 10^{-3}$ & 4.1157 & $0.7660 \times 10^{-4}$ & 4.1188 \\
\hline $2^{-5}$ & $0.1966 \times 10^{-4}$ & 4.0285 & $0.2662 \times 10^{-4}$ & 4.0270 & $0.1902 \times 10^{-4}$ & 4.0273 \\
\hline
\end{tabular}

Table 6: Convergence rates $\mathrm{O}\left(\mathrm{h}^{\theta}+\Delta \mathrm{t}^{\beta}\right)$ for time-split MacCormack by $r_{\mathfrak{u}}^{\mathrm{m}}$, with varying spacing $\mathrm{h}$ and time step $\Delta t$ and $k=h^{2}$.

\begin{tabular}{|c|c|c|c|c|c|c|}
\hline $\mathrm{h}$ & $\||\mathrm{E}(\phi)|\|_{\mathrm{L}^{2}}$ & $\mathrm{r}_{\phi}^{2}$ & $\||\mathrm{E}(\phi)|\|_{\mathrm{L}^{\infty}}$ & $\mathrm{r}_{\phi}^{\infty}$ & $\|\mathrm{E}(\phi)\|_{\mathrm{L}^{1}}$ & $\mathrm{r}_{\phi}^{1}$ \\
\hline $2^{-1}$ & $0.2690 \times 10^{-1}$ & - & $0.3670 \times 10^{-1}$ & - & $0.2590 \times 10^{-1}$ & - \\
\hline $2^{-1}$ & $0.3800 \times 10^{-2}$ & 7.0789 & $0.4900 \times 10^{-2}$ & 7.4898 & $0.3800 \times 10^{-2}$ & 7.8158 \\
\hline $2^{-1}$ & $0.663 \times 10^{18}$ & $0.573 \times 10^{-20}$ & $0.481 \times 10^{19}$ & $0.102 \times 10^{-20}$ & $0.130 \times 10^{18}$ & $0.292 \times 10^{-19}$ \\
\hline
\end{tabular}


The analysis discussed in Section 4, has shown that the numerical scheme is first order convergent in time and fourth order accurate in space. If the result provided in Section 2, page 8, is to believe, this suggests that the time-split MacCormack scheme is inconsistent. Fortunately, we observe from Tests 5.15.3, precisely Figures 1-3, and Tables 1-3, that the three-level time-split MacCormack approach is stable, second order accurate in time and fourth order convergent in space under the time step restriction (2.33), which confirms the theoretical result provided in Section 2, page 8. Thus, the considered method is fast and efficient.
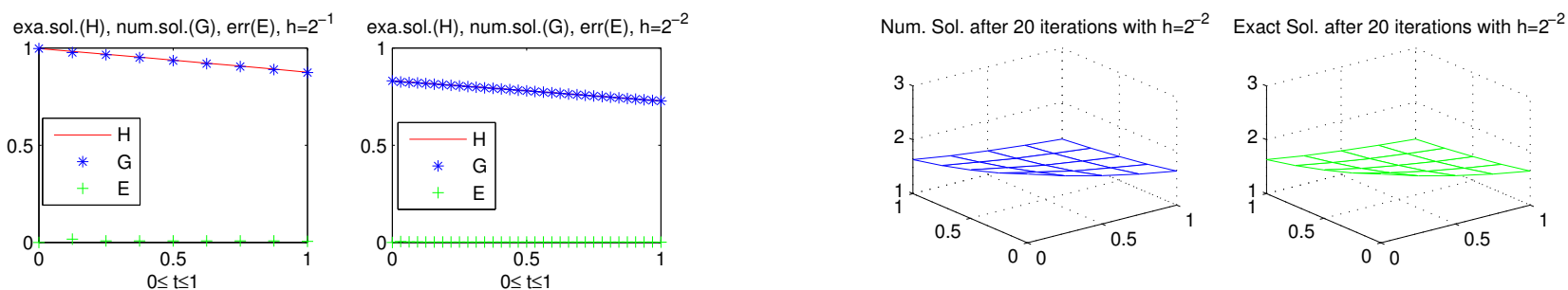

exa.sol.(H), num.sol.(G), err(E), $h=2^{-3}$

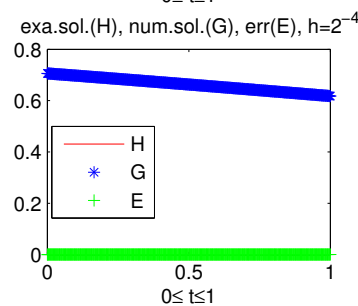

Error after 20 iterations with $\mathrm{h}=2^{-2}$
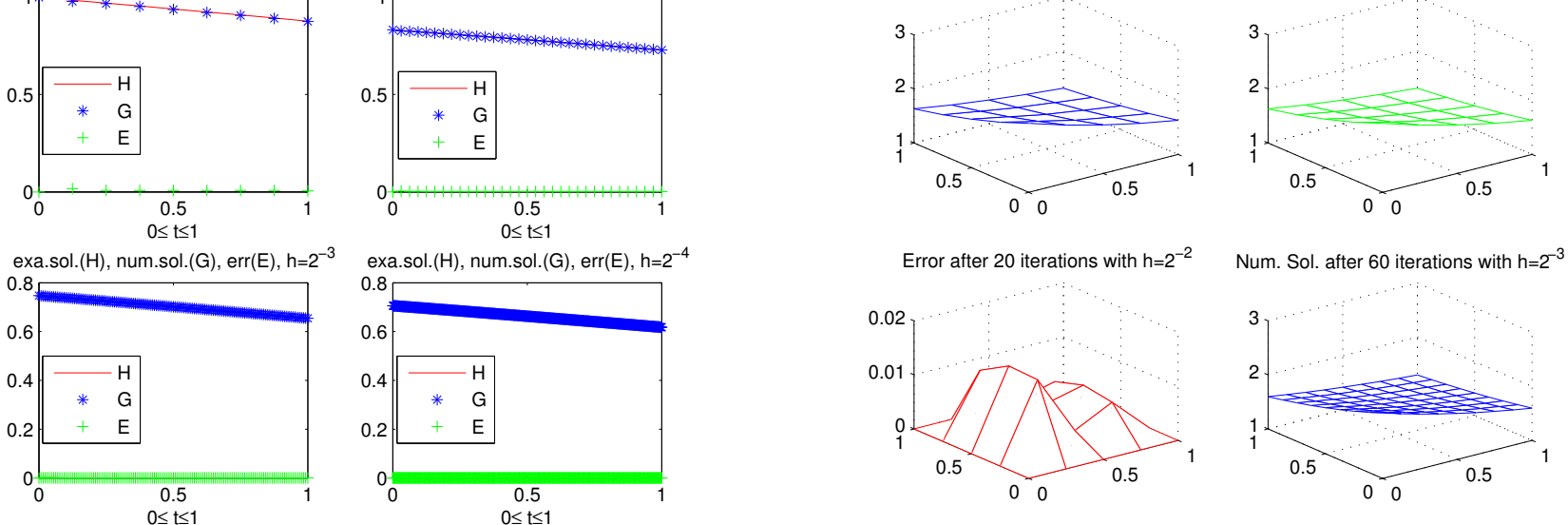

Num. Sol. after 60 iterations with $\mathrm{h}=2^{-3}$

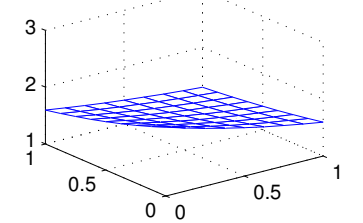

Figure 1: Stability and convergence of a three-level time-split MacCormack method: $a=\mu=1, \bar{\phi}(x, y, t)=1+$ $\exp \left((-1+\sqrt{2}) t-\frac{\sqrt{2}}{2} x-\frac{\sqrt{2}}{2} y\right)$ and $f(\bar{\phi})=2(1-\bar{\phi})$.
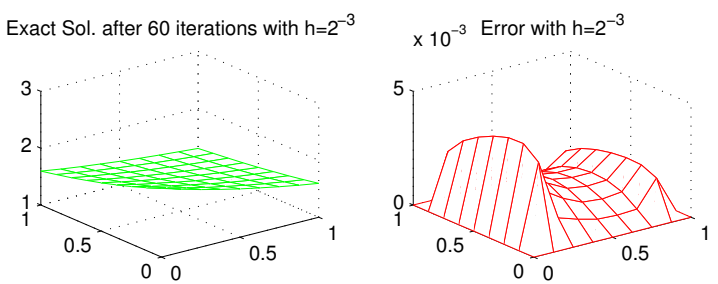

Num. Sol. after 400 iterations with $\mathrm{h}=2^{-4}$ Anal. Sol. after 400 iterations with $\mathrm{h}=2^{-4}$
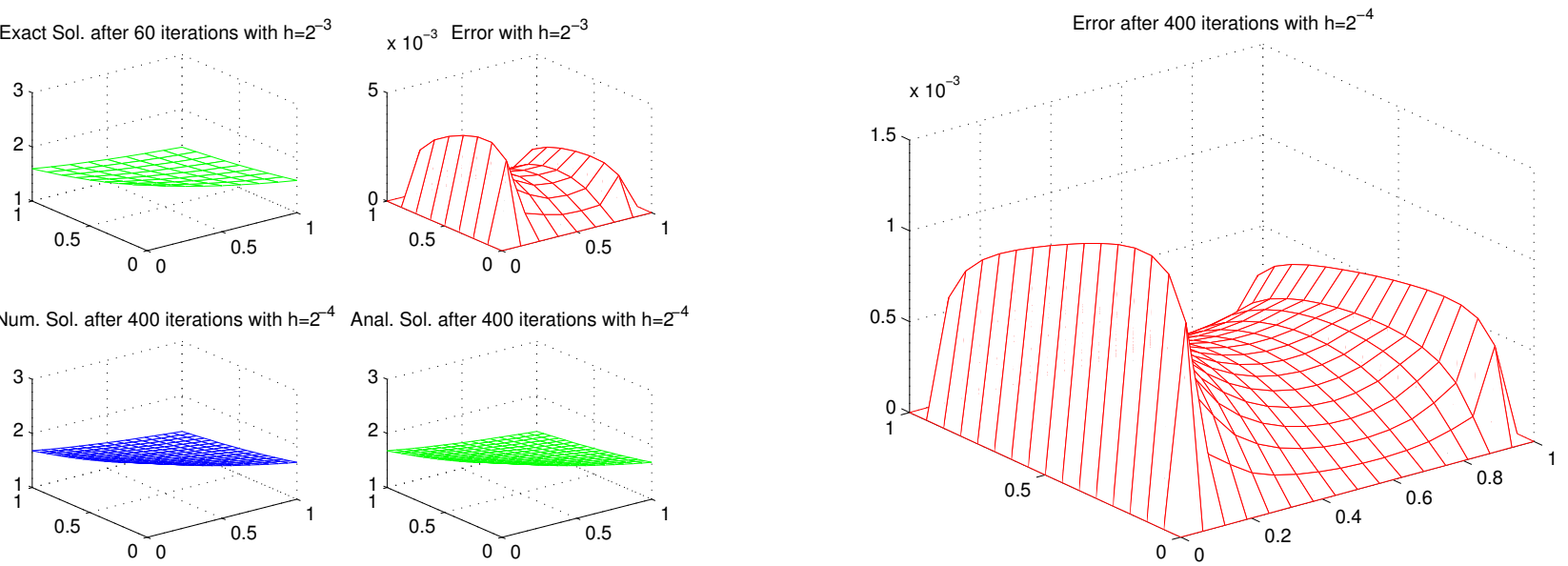

Figure 2: Stability and convergence of a three-level time-split MacCormack approach: $a=\mu=1, \bar{\phi}(x, y, t)=1+$ $\exp \left((-1+\sqrt{2}) t-\frac{\sqrt{2}}{2} x-\frac{\sqrt{2}}{2} y\right)$ and $f(\bar{\phi})=2(1-\bar{\phi})$. 


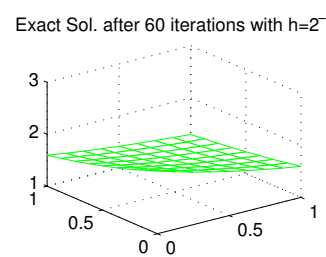

$\times 10^{-3}$ Error with $\mathrm{h}=2^{-3}$

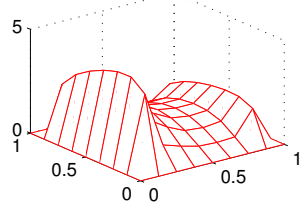

Num. Sol. after 400 iterations with $h=2^{-4}$ Anal. Sol. after 400 iterations with $h=2^{-4}$
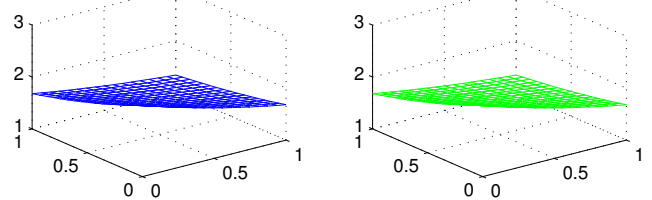

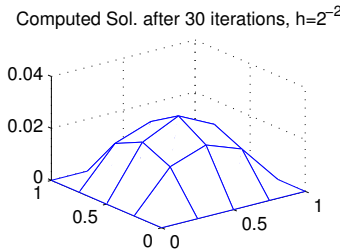

Exact Sol. after 30 iterations, $h=2^{-2}$

$\times 10^{-3}$ Error with $\mathrm{h}=2^{-2}$

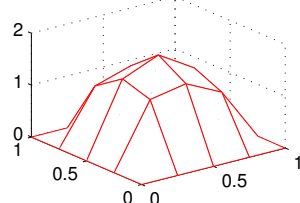

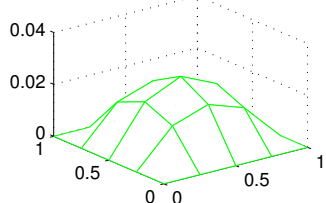

Computed Sol. after 120 iterations with $\mathrm{h}=2^{-3}$

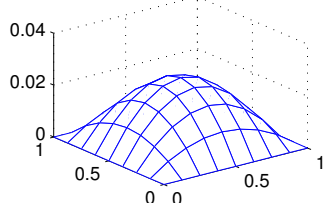

Figure 3: Stability and convergence of a three-level time-split MacCormack method: $a=\mu=1, \bar{\phi}=\exp (-t) x y(1-x)(1-y)$, $f=-\bar{\phi}+\exp (-t)[x(1-x)(3-2 y)+y(1-y)(3-2 x)]$.

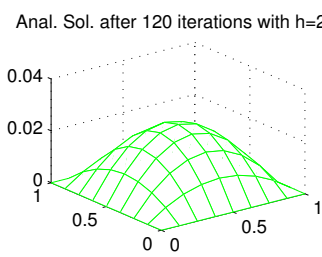

$\times 10^{-4}$ Error with $\mathrm{h}=2^{-3}$

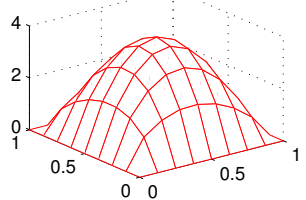

Num. Sol. after 500 iterations with $h=2^{-4}$ Exact Sol. after 500 iterations with $h=2^{-4}$
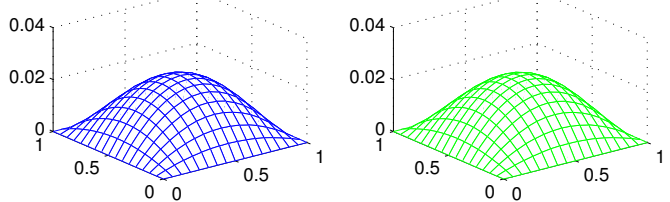

Error after 500 iterations with $\mathrm{h}=2^{-4}$

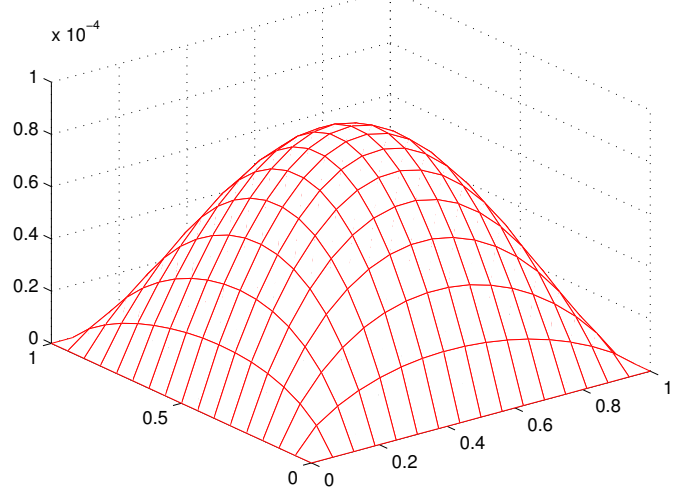

Figure 4: Stability and convergence of a three-level time-split MacCormack method: $a=\mu=1, \bar{\phi}=\exp (-t) x y(1-x)(1-y)$, $f=-\bar{\phi}+\exp (-t)[x(1-x)(3-2 y)+y(1-y)(3-2 x)]$.
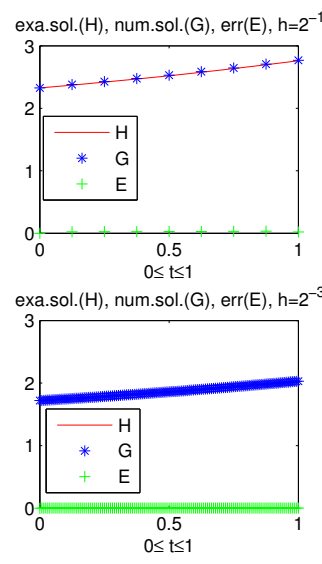

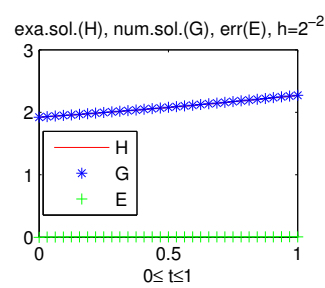

exa.sol.(H), num.sol.(G), err(E), h=2-4

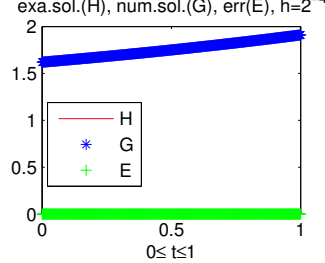

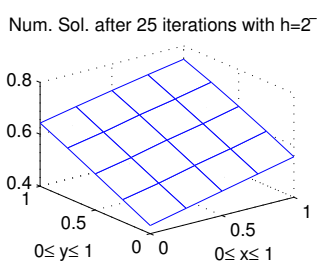

$\times 10^{-3} \quad$ Error

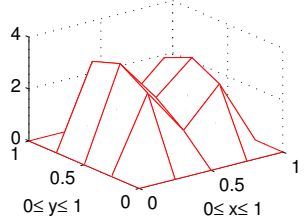

Exact Sol. after 25 iterations, $\mathrm{h}=2^{-2}$

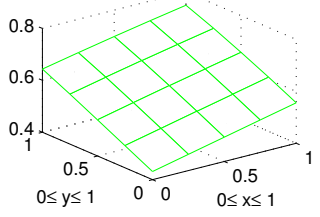

Num. Sol. after 50 iterations with $\mathrm{h}=2^{-3}$

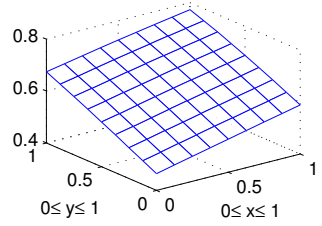

Figure 5: Stability and convergence of a three-level time-split MacCormack method: $a=\mu=1, \bar{\phi}(x, y, t)=$ $\left[1+\exp \left(\left(-1+\frac{\sqrt{3}+1}{2}\right) t-\frac{\sqrt{3}}{2} x-\frac{1}{2} y\right)\right]^{-1}$ and $f(\bar{\phi})=2(1-\bar{\phi}) \bar{\phi}^{2}$. 

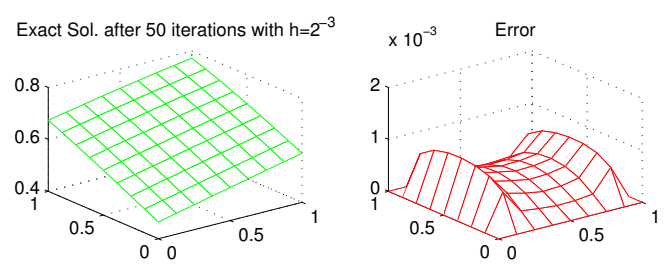

Num. Sol. after 200 iterations with $\mathrm{h}=2^{-4}$ Anal. Sol. after 200 iterations with $\mathrm{h}=2^{-4}$
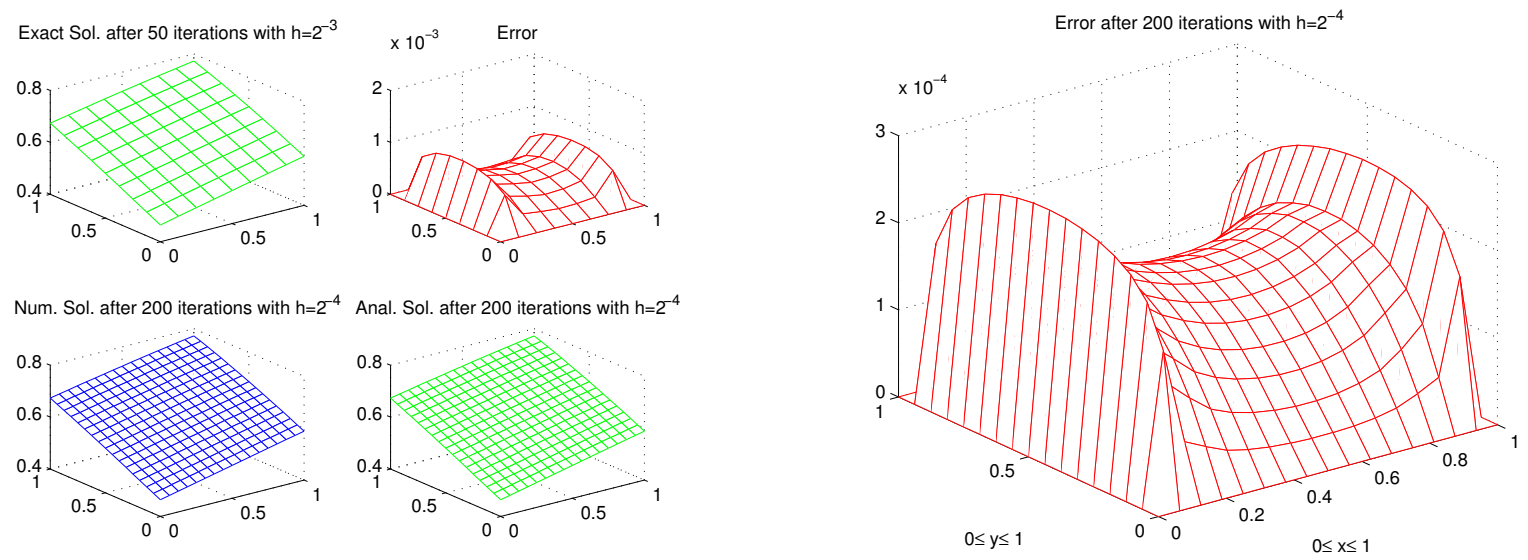

Figure 6: Stability and convergence of a three-level time-split MacCormack method: $a=\mu=1, \bar{\phi}(x, y, t)=$ $\left[1+\exp \left(\left(-1+\frac{\sqrt{3}+1}{2}\right) t-\frac{\sqrt{3}}{2} x-\frac{1}{2} y\right)\right]^{-1}$ and $f(\bar{\phi})=2(1-\bar{\phi}) \bar{\phi}^{2}$.

\section{General conclusion and future works}

In this work, we have presented a detailed analysis of the stability, error estimates and convergence rate of a three-level explicit time-split MacCormack scheme for solving the two-dimensional nonlinear unsteady advection-diffusion equation with constant coefficients (1.1) subjects to suitable initial and boundary conditions (1.2)-(1.3). Our study has shown that the considered method is stable, consistent, second order accurate in time and fourth order convergent in space under the time step requirement (2.33). This theoretical analysis is confirmed by a wide set of numerical evidences (Figures 1-6 and Tables 1-3). Numerical experiments also suggest that the proposed algorithm is: (1) more efficient and effective than a broad range of numerical schemes for solving the initial-boundary value problem (1.1)-(1.3); (2) fast and robust tools for the integration of general systems of hyperbolic/parabolic partial differential equations. Thus, our future works will analyze an approximate solution of the two-dimensional unsteady advection-diffusion equation with sink/source terms using the new approach.

\section{Acknowledgment}

This work has been partially supported by the Deanship of Scientific Research of Al-Imam Muhammad Ibn Saud Islamic University (IMSIU) under the Grant No. 331203.

\section{References}

[1] F. A. Anderson, R. H. Pletcher, J. C. Tannehill, Computational fluid mechanics and Heat Transfer, Second Edition, Taylor and Francis, New York, (1997). 2

[2] M. I. Asenso, B. Ayuso, L. Ferragut, G. sangalli, Numerical methods for modelling leaching of polluants in soils, Adv. Eng. Software, 38 (2007), 429-438. 1

[3] R. Codina, J. Blasco, Analysis of a stabilized finite element approximation of the transient convection-diffusion-reaction equation using orthogonal subscales, Comput. Visual. Sci., 4 (2002), 167-174. 1

[4] R. Codina, J. Principe, C. Munoz, J. Baiges, Numerical modelling of chlorine concentration in water storage tanks, Int. J. Numer. Methods Fluids, 79 (2015), 84-107. 1

[5] M. J. Crochet, A. R. Davies, K. Walters, Numerical simulations of non-Newtonian flow, Elsevier, New York, (1984). 1

[6] J. de Frutos, A. B. Garcia-Archilla, V. John, J. Novo, An adaptive SUPG method for evolutionary convection-diffusion equations, Comput. Methods Appl. Mech. Engrg., 273 (2014), 219-237. 1

[7] J. de Frutos, A. B. Garcia-Archilla, J. Novo, Local error estimates for the SUPG method applied to evolutionary convectionreaction-diffusion equations J. Sci. Comput., 66 (2016), 528-554. 1

[8] J. Douglas, T. F. Russell, Numerical methods for convection-dominated diffusion problems based on combining the method of characteristics with finite element or finite difference procedures, SIAM J. Numer. Anal., 19 (1982), 871-885. 1 
[9] I. Harari, T. J. R. Hughes, Finite element methods for the Helmholtz equation in an exterior domain: model problem, Comput. Methods Appl. Mech. Engrg., 87 (1991), 59-96. 1

[10] M. A. Hossain, Modeling advective-dispersive transport with reaction: an accurate explicit finite difference model, Appl. Math. Comput., 102 (1999), 101-108.

[11] A. Huerta, J. Donea, Time-accurate solution of stabilized convection-reaction-diffusion equations, Comm. Numer. Methods Engrg., 18 (2002), 565-573. 1

[12] W. Jaäger, Y. G. Lu, Global regularity of solutions for general degenerate parabolic equations in one-dimensional, J. Differential Equations, 140 (1997), 365-377. 1

[13] V. John, J. Novo, On (essentially) non-oscillatory discretizations of evolutionary convection-diffusion equations, J. Comput. Phys., 231 (2012), 1570-1585. 1

[14] P. Lax, B. Wendroff, Systems of conservation laws, Comm. Pure Appl. Math., 13 (1960), 217-237. 1

[15] L. Leboucher, Monotone scheme and boundary conditions for finite volume simulation of magnetohynamic internal flows at high Hartmann number, J. Comput. Phys., 150 (1999), 181-198. 1

[16] Q. Lingzhi, C. Huiping, G. Rui, F. Xinlong, The characteristic variational muliscale method for convection-dominated convection-diffusion-reaction problems, Int. J. Heat Mass Transfer, 572 (2014), 461-469. 5, 5.3

[17] Y. G. Lu, W. Jäger, On solutions to nonlinear reaction-diffusion-convection equations with degenerate diffusion, J. Differential Equations, 170 (2001), 1-21. 1

[18] R. W. MacCormack, An efficient numerical method for solving the time-dependent compressible Navier-Stokes equations at high Reynolds numbers, NASA TM, 1976 (1976), 73-129. 1

[19] R. W. MacCormack, A numerical method for solving the equations of compressible viscous flows, AIAA J., 20 (1982), 1275-1281. 1

[20] R. W. MacCormack, A. J. Paullay, The effect of viscosity in hyrvelocity impact cratering, AIAA paper, American Institute of Aeronautics and astrophysics, 69 (1969), 416-422.

[21] R. W. MacCormack, A. J. Paullay, Computational efficiency achieved by time splitting of finite difference operators, In: 10th Aerospace Sciences Meeting, 1972 (1972), 72-154. 1, 2

[22] C. Man, C. W. Tsai, A high order predictor-corrector scheme for two-dimensional advection-diffusion equation, Int. J. Numer. Methods Fluids, 56 (2008), 401-418. 1

[23] F. T. Namio, E. Ngondiep, R. Ntchantcho, J. C. Ntonga, Mathematical Model of Complete Shallow Water Problem with Source Terms, Stability Analysis of Lax-Wendroff Scheme, J. Theor. Comput. Sci., 2 (2015), 12 pages. 1

[24] E. Ngondiep, Asymptotic growth of the spectral radii of collocation matrices approximating elliptic boundary problems, Int. J. Appl. Math. Comput., 4 (2012), 199-219. 1

[25] E. Ngondiep, Long Time Stability and Convergence Rate of MacCormack Rapid Solver Method for Nonstationary StokesDarcy Problem, Comput. Math. Appl., 75 (2018), 3663-3684.

[26] E. Ngondiep, A novel three-level time-split MacCormack method for solving two-dimensional viscous coupled Burgers' equations, arXiv, 2019 (2019), 17 pages.

[27] E. Ngondiep, A three-level explicit time-split MacCormack method for $2 D$ nonlinear reaction-diffusion equations, arXiv, 2019 (2019), 25 pages. 2, 2, 2, 4

[28] E. Ngondiep, Error estimate of MacCormack rapid solver method for $2 D$ incompressible Navier-Stokes problems, arXiv, 2019 (2019), 26 pages.

[29] E. Ngondiep, Long time unconditional stability of a two-level hybrid method for nonstationary incompressible Navier-Stokes equations, J. Comput. Appl. Math., 345 (2019), 501-514.

[30] E. Ngondiep, Stability analysis of MacCormack rapid solver method for evolutionary Stokes-Darcy problem, J. Comput. Appl. Math. 345 (2019), 269-285. 1

[31] E. Ngondiep, An efficient explicit approach for predicting the Covid-19 spreading with undetected infectious: The case of Cameroon, arXiv, 2020 (2020), 26 pages. 1

[32] E. Ngondiep, An efficient three-level explicit time-split scheme for solving two-dimensional unsteady nonlinear coupled Burgers equations, Internat. J. Numer. Methods Fluids, 92 (2020), 266-284. 1, 2, 2

[33] E. Ngondiep, An efficient three-level explicit time-split approach for solving two-dimensional heat conduction equation, Appl. Math. Inf. Sci., 14 (2020), 1075-1092. 1

[34] E. Ngondiep, A fourth-order two-level factored implicit scheme for solving two-dimensional unsteady transport equation with time dependent dispersion coefficients, Int. J. Comput. Methods Eng. Sci. Mech., 22 (2021), 253-264. 1

[35] E. Ngondiep, A novel three-level time-split MacCormack scheme for two-dimensional evolutionary linear convectiondiffusion-reaction equation with source term, Int. J. Comput. Math., 98 (2021), 47-74. 1

[36] E. Ngondiep, A robust three-level time-split MacCormack scheme for solving two-dimensional unsteady convectiondiffusion equation, J. Appl. Comput. Mech., 7 (2021), 559-577.

[37] E. Ngondiep, A two-level factored Crank-Nicolson method for two-dimensional nonstationary advection-diffusion equation with time dependent dispersion coefficients and source/sink term, Adv. Appl. Math. Mech., 13 (2021), 1005-1026. 1

[38] E. Ngondiep, A robust numerical two-level second-order explicit approach to predicting the spread of Covid-2019 pandemic with undetected infectious cases, J. Comput. Appl. Math., 403 (2022), 13 pages. 1

[39] E. Ngondiep, R. Alqahtani, J. C. Ntonga, Stability analysis and convergence rate of MacCormack scheme for complete Shallow water equations with source terms, arXiv, 2019 (2019), 27 pages. 1 
[40] E. Ngondiep, N. Kerdid, M. A. M. Abaoud, I. A. I. Aldayel, A three-level time-split MacCormack method for twodimensional nonlinear reaction-diffusion equations, Internat. J. Numer. Methods Fluids, 92 (2020), 1681-1706. 1

[41] T. Roose, S. J. Chapman, P. K. Maini, Mathematical models of a vascular tumor growth, SIAM Review, 49 (2007), 179-208. 1

[42] T. E. Tezduyar, Y. J. Park, H. A. Deans, Finite element procedures for time-dependent convection-diffusion-reaction systems, Int. J. Numer. Methods Fluids, 7 (1987), 1013-1033. 1

[43] W. P. Ziemer, Interior and boundary continuity of weak solutions of degenerate parabolic equations, Trans. Amer. Math. Soc., 271 (1982), 733-748. 1 